\title{
Regularization, renormalization and consistency conditions in QED with $x$-electric potential steps
}

\author{
S. P. Gavrilov ${ }^{1,3, a}$, D. M. Gitman ${ }^{1,2,4, b}$ \\ ${ }^{1}$ Department of Physics, Tomsk State University, 634050 Tomsk, Russia \\ 2 P.N. Lebedev Physical Institute, 53 Leninskiy Prospect, 119991 Moscow, Russia \\ ${ }^{3}$ Department of General and Experimental Physics, Herzen State Pedagogical University of Russia, Moyka Embankment 48, 191186 St. Petersburg, \\ Russia \\ ${ }^{4}$ Institute of Physics, University of São Paulo, CP 66318, São Paulo, SP CEP 05315-970, Brazil
}

Received: 23 May 2020 / Accepted: 10 August 2020 / Published online: 4 September 2020

(c) The Author(s) 2020

\begin{abstract}
The present article is an important addition to the nonperturbative formulation of QED with $x$-steps presented by Gavrilov and Gitman (Phys. Rev. D. 93:045002, 2016). Here we propose a new renormalization and volume regularization procedures which allow one to calculate and distinguish physical parts of different matrix elements of operators of the current and of the energy-momentum tensor, at the same time relating the latter quantities with characteristics of the vacuum instability. For this purpose, a modified inner product and a parameter $\tau$ of the regularization are introduced. The latter parameter can be fixed using physical considerations. In the Klein zone this parameter can be interpreted as the time of the observation of the pair-production effect. In the refined formulation of QED with $x$-steps, we succeeded to consider the back-reaction problem. In the case of an uniform electric field $E$ confined between two capacitor plates separated by a finite distance $L$, we see that the smallness of the back-reaction implies a restriction (the consistency condition) on the product $E L$ from above.
\end{abstract}

\section{Introduction}

The effect of particle creation by strong electromagnetic and gravitational fields has been attracting attention for a long time. The effect has a pure quantum nature and was first considered in the framework of the relativistic quantum mechanics with the understanding that all the questions can be answered only in the framework of quantum field theory (QFT). QFT with external background is to a certain extent an appropriate model for such calculations. In the framework

\footnotetext{
a e-mails: gavrilovsergeyp@yahoo.com; gavrilovsp@herzen.spb.ru

b e-mail: gitman@if.usp.br (corresponding author)
}

of such a model, particle creation is closely related to a violation of the vacuum stability with time. Backgrounds (external fields) that violate the vacuum stability are electric-like fields that are able to produce nonzero work when interacting with charged particles. The following statement of the problem is usually considered. Assuming that the initial state of the quantized field of matter is the vacuum (often called the initial vacuum, or in-vacuum), its evolution with time under the influence of some electric like field of finite length and duration is studied. After the external field is switched off one can define a new vacuum (final vacuum, or out-vacuum) and calculate pairs of particles (out-particles) appearing above this vacuum. In the general case, the out-vacuum differs from the in-one. Depending on the structure of the electric like fields, different approaches for calculating the particle creation effect (Schwinger effect) were proposed and realized. Initially, for simplification of calculations and correspondence with the idealized problem statement described above, the effect of particle creation was considered for uniform time-dependent external electric fields that are switched on and off at the initial and the final time instants, respectively. In what follows, we call such a kind of external field the $t$-electric potential step ( $t$-step in what follows). In such an approach, one can neglect the fact that real in- or outparticles are spatially localized, and represent them by some plane waves. Scattering, particle creation from the vacuum and particle annihilation by the $t$-steps were considered in the framework of the relativistic quantum mechanics; see Refs. [1-5]; a more complete list of relevant publications can be found in $[4,5]$. A general formulation, nonperturbative with respect to the time-dependent external background of quantum electrodynamics (QED), was developed in Refs. [6-8].

It is clear that approaches elaborated for nonperturbative calculations in QED with $t$-step are not applicable to 
the cases of strong electric fields that are concentrated in restricted space areas. However, there exist many physically interesting situations where an external background formally is not switched off. As an example, we may mention a time-independent but non-uniform electric field of a constant direction situated in a region $S_{\text {int }}$ between two planes $x=x_{\mathrm{L}}$ and $x=x_{\mathrm{R}}$; it switches off in macroscopic regions on the left of $x=x_{\mathrm{L}}$ and on the right of $x=x_{\mathrm{R}}$. Namely,

$$
\begin{aligned}
& \mathbf{E}(X)=\mathbf{E}(x)=(E(x), 0, \ldots, 0), \\
& E(x)=\text { const }>0, \quad x \in S_{\text {int }}=\left(x_{\mathrm{L}}, x_{\mathrm{R}}\right), \\
& E(x)=0, \quad x \in S_{\mathrm{L}}=\left(x_{\mathrm{FL}}, x_{\mathrm{L}}\right], \\
& x \in S_{\mathrm{R}}=\left[x_{\mathrm{R}}, x_{\mathrm{FR}}\right), \quad x_{\mathrm{FL}}<x_{\mathrm{L}}<0, \quad x_{\mathrm{FR}}>x_{\mathrm{R}}>0 .
\end{aligned}
$$

We note that the points $x_{\mathrm{FL}}$ and $x_{\mathrm{FR}}$ are separated from the origin by macroscopic but finite distances. Fields of the latter form represent a kind of spatial or, as we call them conditionally, $x$-electric potential step ( $x$-step in what follows), ${ }^{1}$

$A^{\mu}(X)=\left(A^{0}(x), A^{j}=0, j=1,2, \ldots, D\right), \quad x=X^{1}$.

Further, for convenience, we use the following notation: $U(x)=-e A_{0}(x)$. The magnitude of the $x$-step is

$$
\begin{gathered}
\Delta U=U_{\mathrm{R}}-U_{\mathrm{L}}>0, \quad U_{\mathrm{R}}=-e A_{0}\left(x_{\mathrm{FR}}\right), \\
U_{\mathrm{L}}=-e A_{0}\left(x_{\mathrm{FL}}\right) .
\end{gathered}
$$

Without loss of generality, we choose a gauge $U_{\mathrm{R}}=-U_{\mathrm{L}}$ which emphasizes the existing symmetry between electrons and positrons. In the case when we deal with a one-particle problem and relativistic quantum mechanics is applicable (the case of a noncritical step, $\Delta U<2 m$ ) such a timeindependent approximation of external fields is quite common. The critical $x$-step, $\Delta U>2 m$, creates particles from the vacuum (the Klein paradox is closely related to this process [9-12]) and then has to be considered in the framework of QED. Some heuristic calculations of the particle creation by $x$-steps in the framework of the relativistic quantum mechanics were presented by Nikishov in Refs. [2,13] and later developed in Refs. [14,15]. One should also mention Damour's work [16], which contributed significantly in applying semiclassical methods for treating strong-field problems in astrophysics. Using Damour's approach, mean number of pairs created by a strong uniform electric field confined between two capacitor plates separated by a finite

\footnotetext{
${ }^{1}$ Potentials of an external electromagnetic field $A^{\mu}(X)$ in $d=D+1$ dimensional Minkowski space-time parametrized by coordinates $X=$ $\left(X^{\mu}, \mu=0,1, \ldots, D\right)=(t, \mathbf{r}), X^{0}=t, \mathbf{r}=\left(X^{1}, \ldots, X^{D}\right)$. We assume that the basic Dirac particle is an electron with the mass $m$ and the charge $-e, e>0$, and the positron is its antiparticle. The electric field under consideration accelerates electrons along the $x$ axis in the negative direction and positrons along the $x$ axis in the positive direction.
}

distance was calculated in Ref. [17]. A detailed historical review can be found in Refs. $[14,18,19]$. At that time, however, no justification for such calculations from the QFT point of view was known. This was because exact solutions of relativistic equations (both the Dirac and the Klein-Gordon equations) with an $x$-step are known in the form of stationary plane waves with given longitudinal momenta in the regions $S_{\mathrm{L}}$ and $S_{\mathrm{R}}$. And it is a nontrivial problem how to use such solutions to construct initial and final vacua that are global states determined on a $t$-const hyperplane.

In our recent paper [20] we have presented a technique in the framework of standard strong-field QED that allows one to calculate effects of the vacuum instability in the presence of $x$-steps nonperturbatively. Our approach is based on the following considerations. Physically it makes sense to believe that the field of an $x$-step, given by Eq. (1.1), should be considered as a part of a time-dependent inhomogeneous electric field $\mathbf{E}_{\text {pristine }}(X)$ directed along the $x$-direction, which was switched on very fast before the time instant $t_{\text {in }}$; by this time it had time to spread to the whole area $S_{\text {int }}$ and disappear in the macroscopic regions $S_{\mathrm{L}}$ and $S_{\mathrm{R}}$. Then it was switched off very fast just after the time instant $t_{\text {out }}=t_{\text {in }}+T$. The field $\mathbf{E}_{\text {pristine }}(X)=\mathbf{E}(x)$ in the regions $S_{\mathrm{L}}, S_{\text {int }}$ and $S_{\mathrm{R}}$ from $t_{\text {in }}$ till $t_{\text {out }}$, that is, it acts as a constant field concentrated in a restricted space area $S_{\text {int }}$ (we assume standard volume regularization with respect of hypersurface orthogonal to the $x$ direction) during the sufficiently large (macroscopic) period of time $T$,

$T \gg(e E)^{-1 / 2} \max \left\{1, m^{2} / e E\right\}$,

where $E$ is the average value of the constant field. By analogy with how this is done in the time-independent potential scattering due to noncritical steps, it is assumed that there exist time-independent observables in the presence of critical $x$-steps. For example, it seems quite natural that the pair-production rate and the flux of created particles are constant during the time $T$. It means that a leading contribution to the number density of created electron-positron pairs is assumed to be proportional to the large dimensionless parameter $\sqrt{e E} T$ and is independent from fast switching-on and -off if this parameter satisfies Eq. (1.4). This assumption seems quite natural, as much of this behavior is observed for results obtained in exactly solvable cases with $t$-steps [2126] and numerical calculations; see, e.g. [26]. ${ }^{2}$ It is clear that the process of pair creation is transient. Nevertheless, the condition of the smallness of back-reaction shows there is a window in the parameter range of $E$ and $T$ where the constant field approximation is consistent [28].

\footnotetext{
${ }^{2}$ Note that the pair-production rate per unit volume due to homogeneous fields $\left(x_{\mathrm{L}} \rightarrow-\infty, x_{\mathrm{R}} \rightarrow \infty\right.$ ) of given average intensity is equal to or higher than that for the case of a finite width $x_{R}-x_{L}$; see Ref. [27].
} 
It is well known that in general the vacuum instability is manifest in the fact that the initial (true) vacuum, $\mid 0$, true in $\rangle$, in which the system was before turning on the electric field, differs significantly from the final (true) vacuum, $\mid 0$, true out $\rangle$, in which the system appears after turning off this field. For example, this difference can be estimated considering vacuum mean values of the longitudinal current operator $J^{1}(X)$ in the regions $S_{\mathrm{L}}$ and/or $S_{\mathrm{R}}$ at any time instant $t_{0}$ from $t_{\text {in }}$ till $t_{\text {out }}$. One can calculate the density of the longitudinal current corresponding to final particles as follows:

$$
\begin{aligned}
\bar{J}_{\mathrm{cr}}^{1}= & \left.J_{\mathrm{cr}}^{1}(X)\right|_{t=t_{0}, x \in S_{\mathrm{L} / \mathrm{R}}}, \\
J_{\mathrm{cr}}^{1}(X)= & \left\langle 0, \text { true in }\left|J^{1}(X)\right| 0, \text { true in }\right\rangle \\
& \quad-\left\langle 0, \text { true out }\left|J^{1}(X)\right| 0, \text { true out }\right\rangle .
\end{aligned}
$$

However, both fast switching-on and -off produce electronpositron pairs with quantum numbers of the tiny range of kinetic energy and one can neglect these contributions to any total characteristics of particle creation, which are determined by the sum over all kinetic energies, if the time $T$ satisfies Eq. (1.4) [21-26]. These total characteristics are, for example, the vacuum-to-vacuum transition probability, pairproduction rate, and fluxes of charge and energy of created particles. The mean values given by Eq. (1.5) depend rather weakly on switching-on and -off effects. Neglecting effects of fast switching-on and -off for the total characteristics, one can approximate these quantities using in calculations instead of the true vacua $\mid 0$, true in $\rangle$ and $\mid 0$, true out $\rangle$ some timeindependent states $\mid 0$, in $\rangle$ and $\mid 0$, out $\rangle$, respectively, closest to them in a certain sense. We choose these states as states with a minimum kinetic energy with respect to any uncharged quasistationary state (kinetic energies of the states $\mid 0$, in $\rangle$ and $\mid 0$, out $\rangle$ are the same; see Ref. [20] for details) and the mean values

$$
\begin{aligned}
\left\langle J^{1}\right\rangle_{\text {in }} & =\left.\left\langle 0, \text { in }\left|J^{1}(X)\right| 0, \text { in }\right\rangle\right|_{t=t_{0}, x \in S_{\mathrm{L} / \mathbb{R}}}, \\
\left\langle J^{1}\right\rangle_{\text {out }} & =\left.\left\langle 0, \text { out }\left|J^{1}(X)\right| 0, \text { out }\right\rangle\right|_{t=t_{0}, x \in S_{\mathrm{L} / \mathbb{R}}}
\end{aligned}
$$

represent the leading contribution to the means of Eq. (1.5). The quantities (1.6) are independent from fast switchingon and -off. They are proportional to the number density of partial vacuum states excited due to an electric field, that is, they are proportional to the number density of the created electron-positron pairs, and therefore they are proportional to the large parameter $\sqrt{e E} T$. We can estimate the accuracy of the approximation in use as ${ }^{3}$

$$
\left.\left\langle 0 \text {, true in/out }\left|J^{1}(X)\right| 0 \text {, true in/out }\right\rangle\right|_{t=t_{0}, x \in S_{\mathrm{L} / \mathrm{R}}}
$$

\footnotetext{
3 It would be desirable also to provide detailed numerical evaluations for realistic space-time-dependent configurations of external fields (going beyond locally constant field approximations) to test the validity of the developed approach on a quantitative level.
}

$$
=\left\langle J^{1}\right\rangle_{\text {in } / \text { out }}\left[1+O\left((\sqrt{e E} T)^{-1}\right)\right] .
$$

Note that $\left\langle J^{1}\right\rangle_{\text {in }}$ is positive, while $\left\langle J^{1}\right\rangle_{\text {out }}$ is negative and the accuracy of the approximation for $J_{\mathrm{cr}}^{1}$ is the same.

In the following we call the approximating states $\mid 0$, in $\rangle$ and $\mid 0$, out $\rangle$ initial and final vacua, respectively. In this approximation the field $E_{\text {pristine }}(X)$ acts as a constant field of the $x$-step. Kinetic energies of the vacuum states $\mid 0$, in $\rangle$ and $\mid 0$, out $\rangle$ are equal, however, the mean fluxes of charge and energy for these states are quite distinct, which makes it easy to distinguish between them. We consider electron and positron excitations of these vacua as initial and final particles, respectively. To define the vacua $\mid 0$, in $\rangle$ and $\mid 0$, out $\rangle$ and to construct initial and final Fock spaces, it is sufficient to use the exact solutions of the Dirac equation with a $x$ step; see Ref. [20] for details. Using the developed approach [20] we succeeded to calculate global effects (global quantities) of zero order with respect to the radiative interaction, such that the vacuum-to-vacuum transition amplitude $c_{v}$, the mean differential and the total numbers of created particles, and so on for the number of $x$-steps (step between two capacitor plates, Sauter, Klein, and exponential steps); see [27,2931]. However, a number of important technical and principal questions still need to be answered. Among them are questions such as: how can physical information be extracted from average values of quasilocal quantities, for example, from the matrix elements of operators of the current and of the energy-momentum tensor (EMT) with respect to initial and final vacua. To be able to answer these questions in the present article we refine and substantially supplement our constructions Ref. [20].

The constant electric field of a critical $x$-step produces constant fluxes of created from the vacuum final particles during the time interval $T$. These particles are created as electron-positron pairs and leave a field area $S_{\text {int }}$, wherein electrons are emitted to the region $S_{\mathrm{L}}$ and positrons to the region $S_{\mathrm{R}}$. In these regions the created particles have constant velocities in opposite directions, moving away from the area $S_{\text {int }}$. They form constant longitudinal currents and energy fluxes in the regions $S_{\mathrm{L}}$ and $S_{\mathrm{R}}$ respectively. Since the time interval $T$ and the distances $\left|x_{\mathrm{FL}}-x_{\mathrm{L}}\right|$ and $\left|x_{\mathrm{R}}-x_{\mathrm{FR}}\right|$ are macroscopic, one may believe that, measuring the characteristics of particles in the regions $S_{\mathrm{L}}$ and $S_{\mathrm{R}}$, we are able to evaluate the effect of pair creation in the area $S_{\text {int }}$ for the time interval $T$. To this end, in order for particles created in the area $S_{\text {int }}$ not to leave the regions $S_{\mathrm{L}}$ and $S_{\mathrm{R}}$ in the time $T$, we assume that $\left|x_{\mathrm{FL}}-x_{\mathrm{L}}\right|,\left|x_{\mathrm{R}}-x_{\mathrm{FR}}\right|>T$, i.e., the regions $\left(-\infty, x_{\mathrm{FL}}\right]$ and $\left[x_{\mathrm{FR}},+\infty\right)$ are not causally related to processes in the area $S_{\text {int }}$. In this article we are interested in such quasilocal quantities as the vector of electric current density, $J_{\mathrm{cr}}^{\mu}(x)$, and the energy-momentum tensor of created particles, $T_{\mathrm{cr}}^{\mu \nu}(x)$, measured in the macroscopic regions $S_{\mathrm{L}}$ and $S_{\mathrm{R}}$. In the general case these quantities depend on the 
structure of the electric field, and, in particular, they depend on the coordinate $x$ due to the $x$-dependence of the external field. Our first task is to demonstrate that in the leading term approximation these quantities can be obtained using the vacuum matrix elements of the operators of the current $J^{\mu}(x)$ and of the EMT $T_{\mu \nu}(x)$ with respect to the initial and final vacua defined, according to the general formulation of strong-field QED [6-8] (see also Refs. [3,32]),

$$
\begin{aligned}
& \left\langle J^{\mu}(x)\right\rangle_{\text {in } / \text { out }}=-\left.i e \operatorname{tr}\left[\gamma^{\mu} S_{\text {in/out }}^{c}\left(X, X^{\prime}\right)\right]\right|_{X=X^{\prime}}, \\
& \left\langle\left. J^{\mu}(x)\right|^{c}=-\left.i e \operatorname{tr}\left[\gamma^{\mu} S^{c}\left(X, X^{\prime}\right)\right]\right|_{X=X^{\prime}}\right. \\
& \left\langle T_{\mu \nu}(x)\right\rangle_{\text {in/out }}=\left.i \operatorname{tr}\left[A_{\mu \nu} S_{\text {in/out }}^{c}\left(X, X^{\prime}\right)\right]\right|_{X=X^{\prime}}, \\
& \left\langle T_{\mu \nu}(x)\right\rangle^{c}=\left.i \operatorname{tr}\left[A_{\mu \nu} S^{c}\left(X, X^{\prime}\right)\right]\right|_{X=X^{\prime}}, \\
& A_{\mu \nu}=\frac{1}{4}\left[\gamma_{\mu}\left(P_{\nu}+P_{\nu}^{\prime *}\right)+\gamma_{\nu}\left(P_{\mu}+P_{\mu}^{\prime *}\right)\right],
\end{aligned}
$$

where we use a generalized causal in-out propagator $S^{c}\left(X, X^{\prime}\right)$, and the so-called in-in propagator $S_{\text {in }}\left(X, X^{\prime}\right)$ and out-out propagator $S_{\text {out }}\left(X, X^{\prime}\right)$,

$$
\begin{aligned}
S^{c}\left(X, X^{\prime}\right) & =i\left\langle 0, \text { out }\left|\hat{T} \hat{\Psi}(X) \hat{\Psi}^{\dagger}\left(X^{\prime}\right) \gamma^{0}\right| 0, \text { in }\right\rangle c_{v}^{-1}, \\
c_{v} & =\langle 0, \text { out }| 0, \text { in }\rangle, \\
S_{\text {in }}^{c}\left(X, X^{\prime}\right) & =i\left\langle 0, \text { in }\left|\hat{T} \hat{\Psi}(X) \hat{\Psi}^{\dagger}\left(X^{\prime}\right) \gamma^{0}\right| 0, \text { in }\right\rangle, \\
S_{\text {out }}^{c}\left(X, X^{\prime}\right) & =i\left\langle 0, \text { out }\left|\hat{T} \hat{\Psi}(X) \hat{\Psi}^{\dagger}\left(X^{\prime}\right) \gamma^{0}\right| 0, \text { out }\right\rangle .
\end{aligned}
$$

$\hat{T}$ stands for the chronological ordering operation, $P_{\mu}=$ $i \partial_{\mu}+e A_{\mu}(X), \gamma^{\mu}$ are the $\gamma$-matrices in $d$ dimensions, and the Dirac-Heisenberg operator $\hat{\Psi}(X)$ is assigned to the Dirac field $\psi(X)$ according to conventional QED; see, e.g., [33]. It satisfies the equal time canonical anticommutation relations,

$$
\begin{aligned}
& {\left.\left[\hat{\Psi}(X), \hat{\Psi}\left(X^{\prime}\right)\right]_{+}\right|_{t=t^{\prime}}=0,} \\
& {\left.\left[\hat{\Psi}(X), \hat{\Psi}^{\dagger}\left(X^{\prime}\right)\right]_{+}\right|_{t=t^{\prime}}=\delta\left(\mathbf{r}-\mathbf{r}^{\prime}\right) .}
\end{aligned}
$$

In the general case the introduced matrix elements depend on the coordinate $x$ and thus are quasilocal quantities. Calculating these matrix elements, we meet divergences that indicate the need to use a certain regularization. In the present article we complement the previous constructions presented in Ref. [20], formulating regularization and renormalization procedures for calculating such quasilocal quantities. We express the vector of the electric current density and the EMT of the created particles via properly calculated corresponding vacuum means and consider some important applications of these results. We show that the smallness of the back-reaction implies less restrictive conditions on the electric field in QED with $x$-steps than those that exist for an uniform field.

The article is organized as follows.

In Sect. 2, we recall briefly some basic facts of QED with $x$-steps focusing on details important for treating the above-mentioned problem. We consider such local quantities as vacuum means of operators of the electric current and of the energy-momentum tensor (EMT). These means should be properly regularized and normalized. We propose a new renormalization and volume regularization procedures which allow one to calculate and distinguish physical parts of different matrix elements of operators of the current and of the energy-momentum tensor, at the same time relating the latter with the characteristics of the vacuum instability. In Sect. 3, using properly calculated vacuum mean values of electric current and EMT, we discuss the back-reaction problem. Creating pairs from the vacuum the external electric field is losing its energy and should be depleted over time. Thus, the applicability of the constant field approximation is limited by the smallness of the back-reaction. We study the back-reaction problem considering the case of an uniform electric field confined between two capacitor plates separated by a finite distance $L$. We compare the obtained restrictions with the consistency conditions derived by us in Ref. [28] for QED with $t$-steps. To make the text better readable some features of the solutions of the Dirac equation with critical $x$-steps are put in Appendix A.

We use the relativistic units $\hbar=c=1$ in which the fine structure constant is $\alpha=e^{2} / c \hbar=e^{2}$.

\section{Means values}

\subsection{Regularization}

The basic mathematical tool of the standard strong-field QED consists of the complete sets of exact solutions of relativistic wave equations orthonormalized on a $t$ constant hyperplane. In the framework of the nonperturbative techniques elaborated in Ref. [20] for the strong-field QED with an $x$-step, which acts during the macroscopic time $T$, one can construct such sets with the help of exact solutions of the Dirac equation with an $x$-step. Formally, this technique allows one to express propagators (1.9) via sums over these solutions and obtain explicitly the vacuum matrix elements of operators of the current and of the EMT, defined by Eq. (1.8). These solutions are known in the form of the stationary plane waves with given real longitudinal momenta $p^{\mathrm{L}}$ and $p^{\mathrm{R}}$ in the regions $S_{\mathrm{L}}$ and $S_{\mathrm{R}}$,

$$
\begin{aligned}
& { }_{\zeta} \psi_{n}(X) \sim \exp \left[-i p_{0} t+i \mathbf{p}_{\perp} \mathbf{r}_{\perp}+i p^{\mathrm{L}}\left(x-x_{\mathrm{L}}\right)\right], \quad x \in S_{\mathrm{L}}, \\
& { }^{\zeta} \psi_{n}(X) \sim \exp \left[-i p_{0} t+i \mathbf{p}_{\perp} \mathbf{r}_{\perp}+i p^{\mathrm{R}}\left(x-x_{\mathrm{R}}\right)\right], \quad x \in S_{\mathrm{R}}, \\
& p^{\mathrm{L}}=\zeta \sqrt{\left[\pi_{0}(\mathrm{~L})\right]^{2}-\pi_{\perp}^{2}}, \quad p^{\mathrm{R}}=\zeta \sqrt{\left[\pi_{0}(\mathrm{R})\right]^{2}-\pi_{\perp}^{2}}, \quad \zeta= \pm, \\
& \pi_{0}(\mathrm{~L} / \mathrm{R})=p_{0}-U_{\mathrm{L} / \mathrm{R}}, \quad \pi_{\perp}=\sqrt{\mathbf{p}_{\perp}^{2}+m^{2}} .
\end{aligned}
$$

They are parametrized by a set of quantum numbers $n=$ $\left(p_{0}, p_{\perp}, \sigma\right)$ where $p_{0}$ stands for total energy, $p_{\perp}$ is the 
transversal momentum (the index $\perp$ stands for components of the momentum that are perpendicular to the electric field), and $\sigma$ is the spin polarization. In the case of the critical $x$ steps if $2 \pi_{\perp} \leq \Delta U$ there exist five ranges $\Omega_{k}, k=1, \ldots, 5$, of quantum numbers $n$,

$$
\begin{aligned}
& p_{0} \geq U_{\mathrm{R}}+\pi_{\perp} \Longleftrightarrow \pi_{0}(\mathrm{R}) \geq \pi_{\perp} \quad \text { if } n \in \Omega_{1}, \\
& U_{\mathrm{R}}-\pi_{\perp}<p_{0}<U_{\mathrm{R}}+\pi_{\perp} \text { if } n \in \Omega_{2}, \\
& U_{\mathrm{L}}+\pi_{\perp} \leq p_{0} \leq U_{\mathrm{R}}-\pi_{\perp} \text { if } n \in \Omega_{3}, \\
& U_{\mathrm{L}}-\pi_{\perp}<p_{0}<U_{\mathrm{L}}+\pi_{\perp} \text { if } n \in \Omega_{4}, \\
& p_{0} \leq U_{\mathrm{L}}-\pi_{\perp} \Longleftrightarrow-\pi_{0}(\mathrm{~L}) \geq \pi_{\perp} \text { if } n \in \Omega_{5},
\end{aligned}
$$

where the solutions $\zeta \psi_{n}(X)$ and $\zeta \psi_{n}(X)$ have similar forms and properties for a given $\pi_{\perp}$. In the ranges $\Omega_{2}$ and $\Omega_{4}$ we deal with standing waves completed by linear superpositions of solutions ${ }_{ \pm} \psi_{n}(X)$ and ${ }^{ \pm} \psi_{n}(X)$ with currents that are equal in magnitude. The explicit structure of the solutions $\zeta \psi_{n}(X)$ and ${ }^{\zeta} \psi_{n}(X)$ on the whole axis $x$ is given by Eq. (A2) in Appendix A. It is shown in the framework of QED by using one-particle mean currents and the energy fluxes that, depending on the asymptotic behavior on the regions $S_{\mathrm{L}}$ and $S_{\mathrm{R}}$, the plane waves $\zeta \psi_{n}(X)$ and ${ }^{\zeta} \psi_{n}(X)$ are identified unambiguously as the components of the initial and final wave packets of electrons and positrons; see Sects. V and VII and Appendices C1 and C2 in Ref. [20]. However, unlike the global quantities considered in Ref. [20], the quasilocal quantities (1.8) calculated with the help of such plane waves depend on the volume regularization parameters. That is why in the following we turn to a clarification of the physical meaning of these parameters, refining our regularization construction.

Stationary plane waves of the type (2.1) are usually used in potential scattering theory, where they represent one-particle states with corresponding conserved longitudinal currents. Such an one-particle consideration is valid in all ranges $\Omega_{k}$, except the range $\Omega_{3}$, where the many-particle quantum field consideration is essential. Note that the range $\Omega_{3}$ often is referred to as the Klein zone.

According to a standard volume regularization, we believe that the system under consideration is situated in a large space-time box that has a spatial volume $V_{\perp}$ of the $(d-1)$ dimensional hypersurface orthogonal to the electric field direction $x$ and the time dimension $T$. Both $V_{\perp}$ and $T$ are macroscopically large. It is supposed that all the solutions of the Dirac equation $\psi(X)$ are periodic under transitions from one box to another. Under these suppositions, the matrix elements of a charge transfer across the area $V_{\perp}$ of the $x=$ const hyperplane during the time $T$, given by Eq. (A5) in the Appendix A, do not depend on $x$ and can be used as an inner product on the hyperplane $x=$ const. The plane waves of the type (2.1) are orthonormalized with respect to the inner product (A5). Unlike wave functions of a one-particle theory, the vacuum vectors $\mid 0$, in $\rangle$ and $\mid 0$, out $\rangle$ are global states that are determined over the causally related part of a space volume at a given time instant. To determine these vacua and construct the corresponding in and out states in an adequate Fock space we have to use a time-independent inner product of solutions $\psi(X)$ and $\psi^{\prime}(X)$ of the Dirac equation with the field $E_{\text {pristine }}(X)$ on a $t$ constant hyperplane. We recall that the periodic conditions are not imposed in the $x$ direction. That is why, in contrast to the $t$-step case, the motion of particles in the $x$ direction is unlimited. However, it should be borne in mind that the electric field in question is located inside the region $S_{\text {int }}$ during the time $T$. Consequently, causally related to the area $S_{\text {int }}$ can there be only such parts of the areas $S_{\mathrm{L}}$ and $S_{\mathrm{R}}$, which are located from it at distances not exceeding $c T$. By virtue of this, we propose to refine the volume regularization used in Ref. [20], defining the time-independent inner product on the $t$ constant hyperplane by

$$
\left(\psi, \psi^{\prime}\right)=\int_{V_{\perp}} \mathrm{d} \mathbf{r}_{\perp} \int_{-K^{(\mathrm{L})}}^{K^{(\mathrm{R})}} \psi^{\dagger}(X) \psi^{\prime}(X) \mathrm{d} x,
$$

where an integral over the spatial volume $V_{\perp}$ is completed by an integral over the interval $\left[K^{(\mathrm{L})}, K^{(\mathrm{R})}\right]$ in the $x$ direction. Here $K^{(\mathrm{L} / \mathrm{R})}$ are some macroscopic but finite parameters of the volume regularization, which are in spatial areas where the electric field is absent, $\left|x_{\mathrm{FL}}\right|>K^{(\mathrm{L})} \gg\left|x_{\mathrm{L}}\right|>0$ and $x_{\mathrm{FR}}>K^{(\mathrm{R})} \gg x_{\mathrm{R}}>0$. Such an inner product is timeindependent if solutions obey certain boundary conditions that allow one to integrate by parts in Eq. (2.3) neglecting boundary terms. The standard understanding as regards the volume regularization in QFT that physical states are wave packets that vanish on the remote boundaries is also accepted. The inner product (2.3) is conserved for such states. However, considering solutions of the type (2.1) that are plane wave components of the packets, which do not vanish at the spatial infinity, we must accept some additional assumptions to provide the time independence of the inner product (2.3). First we note that states with different quantum numbers $n$ are independent, therefore decompositions of vacuum matrix elements (1.8) into the plane waves with given $n$ do not contain interference terms; see Appendix A for details. That is why it is enough to consider Eq. (2.3) only for a particular case of plane waves $\zeta \psi_{n}(X)$ and $\zeta \psi_{n}(X)$ with equal $n$. Assuming that the areas $S_{\mathrm{L}}$ and $S_{\mathrm{R}}$ are much wider than the area $S_{\text {int }}$,

$K^{(\mathrm{L})}-\left|x_{\mathrm{L}}\right|, \quad K^{(\mathrm{R})}-x_{\mathrm{R}} \gg x_{\mathrm{R}}-x_{\mathrm{L}}$,

and the potential energy $U(x)$ is a smooth function, the principal value of the integral (2.3) is determined by integrals over the areas $x \in\left[-K^{(\mathrm{L})}, x_{\mathrm{L}}\right]$ and $x \in\left[x_{\mathrm{R}}, K^{(\mathrm{R})}\right]$, where the electric field is zero. Thus, it is possible to evaluate integrals of the form (2.3) for any form of the external field, using only 
the asymptotic behavior (2.1) of functions in the regions $S_{\mathrm{L}}$ and $S_{\mathrm{R}}$ where an electric field is absent and particles are free. The form of the electric field affects only the coefficients $g$ entering into the mutual decompositions of the solutions,

$\eta_{\mathrm{L}}{ }^{\zeta} \psi_{n}(X)=+\psi_{n}(X) g\left(+\left.\right|^{\zeta}\right)-{ }_{-} \psi_{n}(X) g\left(-\left.\right|^{\zeta}\right)$, $\eta_{\mathrm{R} \zeta} \psi_{n}(X)={ }^{+} \psi_{n}(X) g\left(\left.{ }^{+}\right|_{\zeta}\right)-{ }^{-} \psi_{n}(X) g\left(-\left.\right|_{\zeta}\right)$,

where $\eta_{\mathrm{L}}=\eta_{\mathrm{R}}=1$ for $n \in \Omega_{1}, \eta_{\mathrm{L}}=\eta_{\mathrm{R}}=-1$ for $n \in \Omega_{5}$, and $\eta_{\mathrm{L}}=-\eta_{\mathrm{R}}=1$ for $n \in \Omega_{3}$. The coefficients $g^{\prime} \mathrm{s}$ are defined by Eq. (A7) and satisfy the unitary relations (A8) represented in Appendix A. One can see that the norms of the plane waves $\zeta \psi_{n}(X)$ and ${ }^{\zeta} \psi_{n}(X)$ with respect to the inner product (2.3) are proportional to the macroscopically large parameters $\tau^{(\mathrm{L})}=K^{(\mathrm{L})} / v^{\mathrm{L}}$ and $\tau^{(\mathrm{R})}=K^{(\mathrm{R})} / v^{\mathrm{R}}$, where $v^{\mathrm{L}}=\left|p^{\mathrm{L}} / \pi_{0}(\mathrm{~L})\right|>0$ and $v^{\mathrm{R}}=\left|p^{\mathrm{R}} / \pi_{0}(\mathrm{R})\right|>0$ are absolute values of the longitudinal velocities of particles in the regions $S_{\mathrm{L}}$ and $S_{\mathrm{R}}$, respectively; see Sect. IIIC.2 and Appendix B in Ref. [20] for details.

It is shown (see Appendix B in Ref. [20]) that the following couples of plane waves are orthogonal with respect to the inner product (2.3):

$$
\begin{aligned}
& \left({ }_{\zeta} \psi_{n},{ }^{-\zeta} \psi_{n}\right)=0, \quad n \in \Omega_{1} \cup \Omega_{5} ; \\
& \left({ }_{\zeta} \psi_{n},{ }^{\zeta} \psi_{n}\right)=0, \quad n \in \Omega_{3},
\end{aligned}
$$

if the parameters of the volume regularization $\tau^{(\mathrm{L} / \mathrm{R})}$ satisfy the condition

$\tau^{(\mathrm{L})}-\tau^{(\mathrm{R})}=O(1)$,

where $O(1)$ denotes terms that are negligibly small in comparison with the macroscopic quantities $\tau^{(\mathrm{L} / \mathrm{R})}$. One can see that $\tau^{(\mathrm{L})}$ and $\tau^{(\mathrm{R})}$ are macroscopic times of motion of electrons and positrons in the areas $S_{\mathrm{L}}$ and $S_{\mathrm{R}}$, respectively, and they are equal,

$\tau^{(\mathrm{L})}=\tau^{(\mathrm{R})}=\tau$.

It allows one to introduce an unique time of motion $\tau$ for all the particles in the system under consideration. This time can be interpreted as a time of observation of the evolution of the system under consideration.

Thus, there are constructed two linearly independent couples of complete on the $t$-constant hyperplane states with given $n$ that are either "in" or "out" states according to the physical interpretation given in Ref. [20]),

$$
\begin{aligned}
& \text { in }: \psi_{n_{1}}^{(\text {in },+)}=+\psi_{n_{1}}, \psi_{n_{1}}^{(\text {in },-)}=-\psi_{n_{1}} ; \\
& \psi_{n_{5}}^{(\text {in, }+)}={ }^{+} \psi_{n_{5}}, \psi_{n_{5}}^{(\text {in },-)}=-\psi_{n_{5}} ; \\
& \psi_{n_{3}}^{(\text {in },+)}=-\psi_{n_{3}}, \psi_{n_{3}}^{(\text {in, },-)}=-\psi_{n_{3}} ; \\
& \text { out }: \psi_{n_{1}}^{(\text {out },-)}=-\psi_{n_{1}}, \psi_{n_{1}}^{(\text {out },+)}={ }^{+} \psi_{n_{1}} ;
\end{aligned}
$$

$$
\begin{aligned}
& \psi_{n_{5}}^{(\text {out },-)}={ }^{-} \psi_{n_{5}}, \psi_{n_{5}}^{(\text {out },+)}=+\psi_{n_{5}} ; \\
& \psi_{n_{3}}^{\text {(out, }+)}={ }^{+} \psi_{n_{3}} \psi_{n_{3}}^{(\text {out },-)}={ }_{+} \psi_{n_{3}}, n_{k} \in \Omega_{k} .
\end{aligned}
$$

Under condition (2.7) the norms of the plane waves on the $t$-constant hyperplane are

$$
\begin{aligned}
& \left({ }_{\zeta} \psi_{n}, \zeta \psi_{n}\right)=\left({ }^{\zeta} \psi_{n},{ }^{\zeta} \psi_{n}\right)=\mathcal{M}_{n} \text { if } n \in \Omega_{1} \cup \Omega_{3} \cup \Omega_{5}, \\
& \mathcal{M}_{n}=2 \frac{\tau}{T}\left|g\left(+\left.\right|^{+}\right)\right|^{2} \quad \text { if } n \in \Omega_{1} \cup \Omega_{5} \\
& \mathcal{M}_{n}=2 \frac{\tau}{T}\left|g\left(+\left.\right|^{-}\right)\right|^{2} \quad \text { if } n \in \Omega_{3}
\end{aligned}
$$

where the coefficients $g$ are defined by Eq. (A7) in Appendix A.

Note that the plane waves $\zeta \psi_{n}(X)$ and ${ }^{\zeta} \psi_{n}(X)$ coincide with solutions of the Dirac equation, unknown in an explicit form, with the field $E_{\text {pristine }}(X)$ for $t_{\text {in }}<t<t_{\text {out }}$. The time independence of the inner product (2.3) implies that orthonormality relations for the plane waves $\zeta \psi_{n}(X)$ and ${ }^{\zeta} \psi_{n}(X)$ on the $t$-constant hyperplane coincide in fact with orthonormality relations for the solutions of the Dirac equation with the field $E_{\text {pristine }}(X)$ under the corresponding initial or final conditions. In particular, one can construct sets of linearly independent plane waves related to either initial or final particles as follows.

We believe that the sets (2.9) together with solutions $\psi_{n_{2}}$ and $\psi_{n_{4}}$, which are not essential for the problems discussed in this article, form complete in and out sets in the Hilbert space of Dirac spinors at any fixed time instant $t$. This assumption is equivalent to the existence of the propagation function in the space of solutions, which satisfies the standard equal time boundary condition. This fact is crucial for a nonperturbative formulation of QED with $x$-steps.

For convenience of the reader, we recall some basic points of this formulation. One can decompose the Heisenberg operator $\hat{\Psi}(X)$ into the solutions of either initial or final complete sets (2.9) and construct in this way in- and out-states in an adequate Fock space:

$$
\begin{aligned}
& \hat{\Psi}(X)=\sum_{n} \mathcal{M}_{n}^{-1 / 2} \\
& \times\left[A_{n}(\text { in }) \psi_{n}^{(\text {in, },+)}(X)+B_{n}^{\dagger}(\text { in }) \psi_{n}^{(\text {in },-)}(X)\right] \\
&= \sum_{n} \mathcal{M}_{n}^{-1 / 2} \\
& \quad \times\left[A_{n}(\text { out }) \psi_{n}^{(\text {out },+)}(X)+B_{n}^{\dagger}(\text { out }) \psi_{n}^{(\text {out },-)}(X)\right],
\end{aligned}
$$

where operator-valued coefficients are determined with the help of the inner product (2.3),

$$
\begin{aligned}
& A_{n}(\text { in })=\left(\psi_{n}^{(\text {in },+)}, \hat{\Psi}\right), \quad B_{n}^{\dagger}(\text { in })=\left(\psi_{n}^{(\text {in },-)}, \hat{\Psi}\right) \\
& A_{n}(\text { out })=\left(\psi_{n}^{(\text {out },+)}, \hat{\Psi}\right), \quad B_{n}^{\dagger}(\text { out })=\left(\psi_{n}^{(\text {out },-)}, \hat{\Psi}\right)
\end{aligned}
$$


These operators define Fermi annihilation and creation operators for initial or final particles as follows:

$$
\begin{aligned}
& A_{n_{1}} \text { (in) }={ }_{+} a_{n_{1}} \text { (in), } B_{n_{1}}^{\dagger} \text { (in) }={ }^{-} a_{n_{1}} \text { (in); } \\
& A_{n_{1}} \text { (out) }={ }^{+} a_{n_{1}} \text { (out), } B_{n_{1}}^{\dagger} \text { (out) }={ }_{-} a_{n_{1}} \text { (out) ; } \\
& A_{n_{2}} \text { (in) }=A_{n_{2}} \text { (out) }=a_{n_{2}}, B_{n_{2}}^{\dagger} \text { (in) }=B_{n_{2}}^{\dagger} \text { (out) }=0 \text {; } \\
& A_{n_{3}} \text { (in) }={ }^{-} a_{n_{3}} \text { (in), } B_{n_{3}}^{\dagger} \text { (in) }={ }_{-} b_{n_{3}}^{\dagger} \text { (in); } \\
& A_{n_{3}} \text { (out) }={ }^{+} a_{n_{3}} \text { (out), } B_{n_{3}}^{\dagger} \text { (out) }={ }_{+} b_{n_{3}}^{\dagger} \text { (out); } \\
& A_{n_{4}} \text { (in) }=A_{n_{4}} \text { (out) }=0, B_{n_{4}}^{\dagger} \text { (in) }=B_{n_{4}}^{\dagger} \text { (out) }=b_{n}^{\dagger} \text {; } \\
& A_{n_{5}} \text { (in) }={ }^{+} b_{n_{5}}^{\dagger} \text { (in), } B_{n_{5}}^{\dagger} \text { (in) }=-b_{n_{5}}^{\dagger} \text { (in); } \\
& A_{n_{5}} \text { (out) }={ }_{+} b_{n_{5}}^{\dagger} \text { (out), } B_{n_{5}}^{\dagger} \text { (out) }={ }^{-} b_{n_{5}}^{\dagger} \text { (out). }
\end{aligned}
$$

All $a$ and $b$ are annihilation and all $a^{\dagger}$ and $b^{\dagger}$ are creation operators, whereas all $a$ and $a^{\dagger}$ describe electrons and all $b$ and $b^{\dagger}$ describe positrons. All the operators labeled by the argument "in" are interpreted as in-operators, whereas all the operators labeled by the argument "out " are interpreted as out-operators. This identification is confirmed by a detailed analysis in Sects. V, VI, and VII of Ref. [20]. Equations (1.10) yield the standard anticommutation rules for the creation and annihilation in- or out-operators introduced. The unitary transformation (2.5) implies canonical transformations between the in- and out-operators.

The two vacuum vectors $\mid 0$, in $\rangle$ and $\mid 0$, out $\rangle$ are null vectors for all $a$ and $b$ annihilation operators given by Eq. (2.12). The partial vacua in the Fock subspaces with given $n$ are stable only in $\Omega_{i}, i=1,2,4,5$. The electric field violates the vacuum stability in the range $\Omega_{3}$; thus, pair creation occurs only in this range and the total vacuum-to-vacuum transition amplitude $c_{v}$ is formed due to the latter instability. Differential mean numbers of electrons $N_{n}^{a}$ (out) and positrons $N_{n}^{b}$ (out), $n \in \Omega_{3}$, created from the vacuum, are equal, $N_{n}^{b}($ out $)=N_{n}^{a}$ (out) $=N_{n}^{\text {cr }}$, and have the forms

$$
\begin{aligned}
& \left.N_{n}^{a} \text { (out) }=\langle 0, \text { in }|+a_{n}^{\dagger} \text { (out) }{ }^{+} a_{n} \text { (out) } \mid 0, \text { in }\right\rangle \\
& \quad=\left|g\left(-\left.\right|^{+}\right)\right|^{-2}, \\
& \left.N_{n}^{b} \text { (out) }=\langle 0, \text { in }|+b_{n}^{\dagger} \text { (out) }+b_{n} \text { (out) } \mid 0, \text { in }\right\rangle \\
& =\left|g\left(+\left.\right|^{-}\right)\right|^{-2} .
\end{aligned}
$$

The total number of pairs created from the vacuum reads

$$
N^{\mathrm{cr}}=\sum_{n \in \Omega_{3}} N_{n}^{\mathrm{cr}}=\sum_{n \in \Omega_{3}}\left|g\left(-\left.\right|^{+}\right)\right|^{-2} .
$$

A detailed consideration of various physical processes (see Sects. V, VI, and VII and Appendices C and D in Ref. [20]) shows that in the range $\Omega_{1}$ there exist only in- and out-electrons, whereas in the range $\Omega_{5}$ there exist only inand out-positrons. In these ranges electrons and positrons are subject to scattering and reflection only. In the range $\Omega_{2}$ there exist only electrons that are subject to total reflection and have an unbounded motion in the $x \rightarrow-\infty$ direction. In the range $\Omega_{4}$ there exist only positrons that are also subject to total reflection but have an unbounded motion in $x \rightarrow+\infty$ direction. No particle creation in these four ranges is possible. We note that all the rules of the potential scattering theory can be derived in the framework of QFT.

In the range $\Omega_{3}$ the quantum field description of the processes is essential. In this range there exist in- and outelectrons (localizable electron wave packets) that can be situated only to the left of the step, and in- and out-positrons (localizable positron wave packets) that can be situated only to the right of the step. In this range, all the partial vacua with given $n$ are unstable, and particle production from vacuum is possible. These pairs consist of out-electrons and outpositrons that appear on the left and on the right of the step and move there to the left and to the right, respectively. At the same time, the in-electrons that move to the step from the left are subject to the total reflection. After being reflected they move to the left of the step already as out-electrons. Similarly, the in-positrons that move to the step from the right are subject to the total reflection. After being reflected they move to the right of the step already as out-positrons.

In the following the effect of pair production from vacuum is most interesting. In this case electrons and positrons leaving the area $S_{\text {int }}$ enter the areas $S_{\mathrm{L}}$ and $S_{\mathrm{R}}$, respectively. There they continue to move in opposite directions with constant longitudinal velocities $-v^{\mathrm{L}}$ and $v^{\mathrm{R}}$. Then in the range $\Omega_{3}$ the parameter $\tau$ can be interpreted as the observation time of the pair-production process.

\subsection{Renormalization}

Here we complement the above consideration, indicating a procedure that allows one to link quasilocal quantities (1.8) with observable physical quantities specifying the vacuum instability. In the general case, the matrix elements (1.8) contain local contributions due to the vacuum polarization and contributions due to the vacuum instability caused by the external field for all the time $T=t_{\text {out }}-t_{\text {in }}$ of his action. We believe that under the condition (2.4) all local contributions due to the existence of the external field in the area $S_{\text {int }}$ can be neglected. Therefore, it is enough to know the longitudinal currents and energy fluxes through the surfaces $x=x_{\mathrm{L}}$ and $x=x_{\mathrm{R}}$ to construct the initial and final states and to find relations between them and the characteristics of the vacuum instability. It is clear that such fluxes of created pairs depend on the parameter of the volume regularization $\tau$ due to the presence of the normalization factor $M_{n}^{-1 / 2}$ in the field operator decomposition (2.11). Thus, we can find their relation to observable physical quantities and obtain a relation between the parameter $\tau$ and the whole time $T$. Such a relation fixes the proposed renormalization procedure. 
Using the decompositions (2.11) and definitions of the vacua $\mid 0$, in $\rangle$ and $\mid 0$, out $\rangle$, one can find an explicit form of the singular functions (1.9) for all the ranges $\Omega_{k}$; see Sect. VIII in Ref. [20] for details. However, since the partial vacua are stable in the ranges $\Omega_{k}, k=1,2,4,5$, the corresponding contributions from the propagators $S^{c}, S_{\text {in }}^{c}$, and $S_{\text {out }}^{c}$ to the vacuum matrix elements coincide. That is why we do not need explicit forms of the singular functions in these ranges. It is enough to note that using asymptotic behavior of the solutions $\zeta \psi_{n}(X)$ and ${ }^{\zeta} \psi_{n}(X)$ one can verify that contributions due to these ranges to vacuum currents and energy fluxes in the areas $S_{\mathrm{L}}$ and $S_{\mathrm{R}}$ are absent. Similar contributions to the diagonal elements $\left\langle T_{\mu \mu}(x)\right\rangle^{c}$ do not depend on the electric field and have to be neglected according to the standard renormalization procedure. Therefore, contributions due to the propagator $S^{c}$ can affect only the vacuum polarization in the field region $S_{\text {int }}$, the formal substantiation of this fact is not directly related to the issues under consideration and will be considered in a future publication.

We are interested in vacuum instability effects, which are formed exclusively in the Klein zone $\Omega_{3}$. As was mentioned earlier, under condition (2.4) the principal value of integral (2.3) is determined by integrals over the areas $x \in$ $\left[-K^{(\mathrm{L})}, x_{\mathrm{L}}\right]$ and $x \in\left[x_{\mathrm{R}}, K^{(\mathrm{R})}\right]$, where the electric field is zero. This implies that only the areas $S_{\mathrm{L}}$ and $S_{\mathrm{R}}$ determine contributions of the vacuum instability effects to matrix elements (1.8). For this reason, in what follows, we consider these matrix elements only in the areas $S_{\mathrm{L}}$ and $S_{\mathrm{R}}$,

Thus, we may limit ourselves to the contributions to the singular functions that are formed in the Klein zone (we denote such contributions with a tilde at the top),

$$
\begin{aligned}
& \tilde{S}^{c}\left(X, X^{\prime}\right)=\theta\left(t-t^{\prime}\right) \tilde{S}^{-}\left(X, X^{\prime}\right)-\theta\left(t^{\prime}-t\right) \tilde{S}^{+}\left(X, X^{\prime}\right), \\
& \tilde{S}^{-}\left(X, X^{\prime}\right)=i \sum_{n \in \Omega_{3}} \mathcal{M}_{n}^{-1}{ }^{+} \psi_{n}(X) w_{n}(+\mid+){ }^{-} \bar{\psi}_{n}\left(X^{\prime}\right), \\
& \tilde{S}^{+}\left(X, X^{\prime}\right)=i \sum_{n \in \Omega_{3}} \mathcal{M}_{n}^{-1}-\psi_{n}(X) w_{n}(-\mid-)+\bar{\psi}_{n}\left(X^{\prime}\right)
\end{aligned}
$$

$$
\begin{aligned}
& \tilde{S}_{\text {in } / \text { out }}^{c}\left(X, X^{\prime}\right)=\theta\left(t-t^{\prime}\right) \tilde{S}_{\text {in } / \text { out }}^{-}\left(X, X^{\prime}\right) \\
& \quad-\theta\left(t^{\prime}-t\right) \tilde{S}_{\text {in/out }}^{+}\left(X, X^{\prime}\right), \\
& \tilde{S}_{\text {in/out }}^{-}\left(X, X^{\prime}\right)=i \sum_{n \in \Omega_{3}} \mathcal{M}_{n}^{-1} \mp \psi_{n}(X){ }^{\mp} \bar{\psi}_{n}\left(X^{\prime}\right), \\
& \tilde{S}_{\text {in/out }}^{+}\left(X, X^{\prime}\right)=i \sum_{n \in \Omega_{3}} \mathcal{M}_{n}^{-1} \mp \psi_{n}(X) \mp \bar{\psi}_{n}\left(X^{\prime}\right),
\end{aligned}
$$

where $\bar{\psi}_{n}(X)=\psi_{n}^{\dagger}(X) \gamma^{0}$, and the amplitudes $w_{n}$ are

$$
\begin{aligned}
& w_{n}(+\mid+)=g\left(\left.{ }^{+}\right|_{-}\right) g\left(\left(\left.^{-}\right|_{-}\right)^{-1}=g\left(++\left._{+}\right|^{-}\right) g\left(+\left.\right|^{+}\right)^{-1},\right. \\
& w_{n}(-\mid-)=g\left(\left.{ }^{-}\right|_{+}\right) g\left(\left.{ }^{-}\right|_{-}\right)^{-1}=g\left(\left.{ }_{-}\right|^{+}\right) g\left(+\left.\right|^{+}\right)^{-1} .
\end{aligned}
$$

Here $g^{\prime}$ s are given by Eq. (A.7) in Appendix A. Taking into account Eqs. (2.10) and (2.13) one can see that the normalization factor $M_{n}^{-1}$ depends on the differential numbers $N_{n}^{\text {cr }}$, and on the ratio $T / \tau$,

$\mathcal{M}_{n}^{-1}=\frac{T}{2 \tau} N_{n}^{\mathrm{cr}}$ for $n \in \Omega_{3}$.

Then means (1.8) can be expressed via singular functions (2.16) as follows:

$$
\begin{aligned}
& \left\langle J^{\mu}(x)\right\rangle_{\text {in } / \text { out }}=-\frac{i e}{2} \\
& \quad \times\left.\operatorname{tr}\left\{\gamma^{\mu}\left[\tilde{S}_{\text {in/out }}^{-}\left(X, X^{\prime}\right)-\tilde{S}_{\text {in/out }}^{+}\left(X, X^{\prime}\right)\right]\right\}\right|_{X=X^{\prime}}, \\
& \left\langle T_{\mu \nu}(x)\right\rangle_{\text {in/out }}=\frac{i}{2} \\
& \quad \times\left.\operatorname{tr}\left\{A_{\mu \nu}\left[\tilde{S}_{\text {in/out }}^{-}\left(X, X^{\prime}\right)-\tilde{S}_{\text {in/out }}^{+}\left(X, X^{\prime}\right)\right]\right\}\right|_{X=X^{\prime}} .
\end{aligned}
$$

It follows from Eq. (2.19) that due to the cylindrical symmetry of a problem the transversal components of the mean currents and non-diagonal components of mean values of the EMT vanish,

$$
\begin{aligned}
& \left\langle J^{k}(x)\right\rangle_{\text {in }}=\left\langle J^{k}(x)\right\rangle_{\text {out }}=\left\langle J^{k}(x)\right\rangle^{c}=0, \\
& \quad\left\langle T_{0 k}(x)\right\rangle_{\text {in }}=\left\langle T_{0 k}(x)\right\rangle_{\text {out }}=\left\langle T_{0 k}(x)\right\rangle^{c}=0, \quad k \neq 1, \\
& \left\langle T_{l k}(x)\right\rangle_{\text {in }}=\left\langle T_{l k}(x)\right\rangle_{\text {out }}=\left\langle T_{l k}(x)\right\rangle^{c}=0, \\
& \quad k \neq l, k, l=2, \ldots
\end{aligned}
$$

The relations between in-out matrix elements and in-in and out-out means can be written in terms of a singular function $S^{p}\left(X, X^{\prime}\right)$,

$S^{p}\left(X, X^{\prime}\right)=S_{\text {in }}\left(X, X^{\prime}\right)-S^{c}\left(X, X^{\prime}\right)$,

which was found in Ref. [20],

$S^{p}\left(X, X^{\prime}\right)=i \sum_{n \in \Omega_{3}} \mathcal{M}_{n}^{-1} g\left(\left.{ }^{-}\right|_{-}\right)^{-1}{ }_{-} \psi_{n}(X){ }^{-} \bar{\psi}_{n}\left(X^{\prime}\right)$.

These relations have the form

$$
\begin{aligned}
& \left\langle J^{\mu}(x)\right\rangle_{\text {in }}=\left\langle J^{\mu}(x)\right\rangle^{c}+\left\langle J^{\mu}(x)\right\rangle^{p}, \\
& \left\langle T_{\mu \nu}(x)\right\rangle_{\text {in }}=\left\langle T_{\mu v}(x)\right\rangle^{c}+\left\langle T_{\mu v}(x)\right\rangle^{p},
\end{aligned}
$$

where

$$
\begin{aligned}
& \left\langle\left. J^{\mu}(x)\right|^{p}=-\left.i e \operatorname{tr}\left[\gamma^{\mu} S^{p}\left(X, X^{\prime}\right)\right]\right|_{X=X^{\prime}},\right. \\
& \left\langle T_{\mu v}(x)\right\rangle^{p}=\left.i \operatorname{tr}\left[A_{\mu v} S^{p}\left(X, X^{\prime}\right)\right]\right|_{X=X^{\prime}} .
\end{aligned}
$$

As it follows from Eq. (2.22) the quantities (2.24) are formed exclusively in the Klein zone $\Omega_{3}$. Besides, one can see from Eq. (2.20) that only the quantities $\left\langle J^{0}(x)\right\rangle^{p},\left\langle J^{1}(x)\right\rangle^{p}$, and $\left\langle T^{10}(x)\right\rangle^{p}$ and $\left\langle T_{\mu \mu}(x)\right\rangle^{p}$ are nonzero.

It would be interesting and practically important to associate the quantities (2.19) with quantities characterizing 
directly the effect of pair production. Below, this problem is solved for the first time.

It should be noted that in QED with $t$-steps [6-8] Heisenberg operators of physical quantities (for example, the kinetic energy operator of the Dirac field) are obviously time dependent. That is why one can determine contributions of the final particles, using in-in vacuum means, and setting $t \rightarrow \infty$ (which means considering the time instant when the external field is already switched off and all the corresponding effects of the vacuum polarization vanish). In the case under consideration mean values are time independent, therefore this possibility is not available. An alternative procedure is required to determine contributions of the final particles. Such a procedure is proposed below.

In the general case, the means (2.19) contain local contributions of the vacuum polarization and contributions from the vacuum instability caused by the external field during the whole time $T=t_{\text {out }}-t_{\text {in }}$ of its existence. As was already mentioned, due to condition (2.4) all the local contributions due to the presence of an external field in the area $S_{\text {int }}$ can be neglected. Therefore, we believe that it is enough to know the longitudinal currents and the energy fluxes through the surfaces $x=x_{\mathrm{L}}$ and $x=x_{\mathrm{R}}$ to evaluate the contributions of the initial and final states.

Let us consider nonzero longitudinal fluxes in the areas $S_{\mathrm{L}}$ and $S_{\mathrm{R}}$. Using Eq. (A4) in the singular functions (2.16), one can find

$$
\begin{aligned}
& \left\langle J^{1}(x)\right\rangle_{\text {in }}=-\left\langle J^{1}(x)\right\rangle_{\text {out }}=\left\langle J^{1}(x)\right\rangle^{p}=\bar{J}^{1}, \\
& \quad x \in S_{\mathrm{L}} \text { or } S_{\mathrm{R}}, \\
& \bar{J}^{1}=\frac{e}{2} \sum_{n \in \Omega_{3}} \tilde{j}_{n}^{1}, \quad \tilde{j}_{n}^{1}=N_{n}^{\mathrm{cr}}\left(\tau V_{\perp}\right)^{-1} ; \\
& \left\langle T^{10}(x)\right\rangle_{\text {in }}=-\left\langle T^{10}(x)\right\rangle_{\text {out }}=\left\langle T^{10}(x)\right\rangle^{p}, \\
& \left\langle T^{10}(x)\right\rangle^{p}=\left\{\begin{array}{ll}
\bar{T}^{10}(\mathrm{~L})=-\frac{1}{2} \sum_{n \in \Omega_{3}} \pi_{0}(\mathrm{~L}) \tilde{j}_{n}^{1}, & x \in S_{\mathrm{L}} \\
\bar{T}^{10}(\mathrm{R})=\frac{1}{2} \sum_{n \in \Omega_{3}}\left|\pi_{0}(\mathrm{R})\right| \tilde{j}_{n}^{1}, & x \in S_{\mathrm{R}}
\end{array} .\right.
\end{aligned}
$$

Note that the electric current densities (2.25) (as well as all components $\tilde{j}_{n}^{1}$ ) are conserved along the axis $x$, while the energy flux densities $\bar{T}^{10}(\mathrm{~L})$ in the left area and $\bar{T}^{10}(\mathrm{R})$ in the right area of the axis $x$ are different due to the potential energy difference $\Delta U$. These quantities correspond to the areas where the electric field is absent. They are proportional to the factor $N_{n}^{\text {cr }}$ formed due to an electric field situated in a region $S_{\text {int }}$. If the electric field vanishes, $E \rightarrow 0$, in the area $S_{\text {int }}$, then at the same time the pair production vanishes, $N_{n}^{\mathrm{cr}} \rightarrow 0$. That is why the above densities characterize in a sense real particles and why they cannot change after the electric field is switched off.

The normal forms of the operators $J^{1}$ and $T^{10}$ with respect to the out-vacuum are

$$
\begin{aligned}
N_{\text {out }}\left(J^{1}\right) & =J^{1}-\left\langle 0, \text { out }\left|J^{1}\right| 0, \text { out }\right\rangle, \\
N_{\text {out }}\left(T^{10}\right) & =T^{10}-\left\langle 0, \text { out }\left|T^{10}\right| 0, \text { out }\right\rangle .
\end{aligned}
$$

Then, taking into account Eqs. (2.25) and (2.26), we can calculate the densities of the longitudinal current and energy flux corresponding to the final particles as means in the initial vacuum state,

$$
\begin{aligned}
& \tilde{J}_{\text {cr }}^{1}=\left\langle N_{\text {out }}\left(J^{1}\right)\right\rangle_{\text {in }}=\left\langle J^{1}(x)\right\rangle_{\text {in }}-\left\langle J^{1}(x)\right\rangle_{\text {out }} \\
& \quad=2\left\langle J^{1}(x)\right\rangle^{p}=2 \bar{J}^{1} \\
& \left\langle\tilde{T}^{10}(x)\right\rangle_{\text {cr }}=\left\langle N_{\text {out }}\left[T^{10}(x)\right]\right\rangle_{\text {in }} \\
& \quad=\left\langle T^{10}(x)\right\rangle_{\text {in }}-\left\langle T^{10}(x)\right\rangle_{\text {out }}=2\left\langle T^{10}(x)\right\rangle^{p} .
\end{aligned}
$$

The means (2.28) depend on the parameter $\tau$. In the range $\Omega_{3}$ this parameter can be interpreted as the time of observation of the pair production and is related to the time $T$. We suppose that all the measurements are performed during a macroscopic time $T$ when the external field can be considered as constant. In this case, for example, we believe that the longitudinal current of created particles is equal to the flux density $N^{\mathrm{cr}}\left(T V_{\perp}\right)^{-1}$ of the particles times the charge $e$,

$J_{\mathrm{cr}}^{1}=e N^{\mathrm{cr}}\left(T V_{\perp}\right)^{-1}$,

where $N^{\mathrm{cr}}$ is the total number of pairs created from the vacuum during the time $T$, given by Eq. (2.14). ${ }^{4}$ One can see that $\tilde{J}_{\text {cr }}^{1}$ given by Eq. (2.28) coincides with the physically motivated expression (2.29) under the condition that the times $\tau$ and $T$ coincide, i.e., $\tau=T$. Such a relation fixes the proposed renormalization procedure.

Thus, we have

$$
\begin{aligned}
& J_{\mathrm{cr}}^{1}=2\left\langle J^{1}(x)\right\rangle^{p}=e \sum_{n \in \Omega_{3}} j_{n}^{1}, \quad j_{n}^{1}=N_{n}^{\mathrm{cr}}\left(T V_{\perp}\right)^{-1} ; \\
& \left\langle T^{10}(x)\right\rangle_{\mathrm{cr}}=2\left\langle T^{10}(x)\right\rangle^{p} \\
& \quad= \begin{cases}T_{\mathrm{cr}}^{10}(\mathrm{~L})=-\sum_{n \in \Omega_{3}} \pi_{0}(\mathrm{~L}) j_{n}^{1}, & x \in S_{\mathrm{L}} \\
T_{\mathrm{cr}}^{10}(\mathrm{R})=\sum_{n \in \Omega_{3}}\left|\pi_{0}(\mathrm{R})\right| j_{n}^{1}, & x \in S_{\mathrm{R}} .\end{cases}
\end{aligned}
$$

Here $j_{n}^{1}$ is the flux density of particles created with given $n$. The density $j_{n}^{1}$ and longitudinal current density $J_{\mathrm{cr}}^{1}$ are conserved in the $x$-direction., The density $j_{n}^{1}$ is formed by electrons on the left of the area $S_{\text {int }}$, whereas it is formed by positrons on the right of the area $S_{\text {int }}$. Differential mean numbers of electrons and positrons from pairs with a given $n$ are equal. The differential numbers $N_{n}^{\text {cr }}$ characterize electrons created on the left of the area $S_{\text {int }}$ and at the same time characterize positrons created on the right of the area $S_{\text {int }}$. Thus, on the left of the area $S_{\text {int }}$ and on the right of the area $S_{\text {int }}$ there are fluxes consisting of all electrons and all positrons

\footnotetext{
${ }^{4}$ Note that contributions to the sum (2.14) due to the tiny ranges of low velocities, $v^{\mathrm{L} / \mathrm{R}} \sim\left|x_{\mathrm{L} / \mathrm{R}}\right| / T$, are negligible if the time $T$ satisfies Eq. (1.4). In the following we assume that these subranges are excluded from sums over the range $\Omega_{3}$ and the condition (2.4) is satisfied for any $v^{\mathrm{L} / \mathrm{R}}$ under consideration.
} 
created from vacuum by the external electric field, which, on the one hand, is natural due to physical considerations, and on the other hand confirms the correctness of the applied approach.

Using Eqs. (A4), (2.16), and (2.19), we find that averaging over the in- and out-vacuum of the charge and diagonal components of EMT operators gives the same results,

$$
\begin{aligned}
\left\langle J^{0}(x)\right\rangle_{\text {in }} & =\left\langle J^{0}(x)\right\rangle_{\text {out }}, \\
\left\langle T^{\mu \mu}(x)\right\rangle_{\text {in }} & =\left\langle T^{\mu \mu}(x)\right\rangle_{\text {out }} \text { if } x \in S_{\mathrm{L}} \text { or } S_{\mathrm{R}} .
\end{aligned}
$$

This coincidence (at least for a time) is an important distinguishing feature of the in and out states in the theory under consideration. In particular, the characteristics of the vacuum states do not change over time $T$. However, the initial and final vacua differ due to the corresponding fluxes of charges and energy through the surface $x=$ const.

One can see how quantities (2.32) are related to the direct characteristics of the vacuum instability. To this end, in these quantities, we separate the contributions of the matrix elements $\left\langle J^{\mu}(x)\right\rangle^{c}$ and $\left\langle T_{\mu \nu}(x)\right\rangle^{c}$ given in Eq. (1.8) on the left of the area $S_{\text {int }}$ and on the right of the area $S_{\text {int }}$. Using the representations (2.16), one can see that $\tilde{S}^{-}\left(X, X^{\prime}\right)$ on the area $x \in S_{\mathrm{R}}$ and $\tilde{S}^{+}\left(X, X^{\prime}\right)$ on the area $x \in S_{\mathrm{L}}$ represent $x$-dependent interference terms, containing factors $\sim$ $\exp \left[i 2\left|p^{\mathrm{R}}\right|\left(x-x_{\mathrm{R}}\right)\right]$ and $\exp \left[-i 2\left|p^{\mathrm{L}}\right|\left(x-x_{\mathrm{L}}\right)\right]$, respectively. Such terms do not contribute to the fluxes $\left\langle J^{1}(x)\right\rangle^{c}$ and $\left\langle T^{10}(x)\right\rangle^{c}$. They do not contribute to any integrals over $x$ and, therefore, they can be neglected in the densities $\left\langle J^{0}(x)\right\rangle^{c}$ and $\left\langle T^{\mu \mu}(x)\right\rangle^{c}$. Now we analyze the opposite case, namely, let us consider representations of the function $\tilde{S}^{-}\left(X, X^{\prime}\right)$ in the area $x \in S_{\mathrm{L}}$ and of the function $\tilde{S}^{+}\left(X, X^{\prime}\right)$ on the area $x \in S_{\mathrm{R}}$. Using Eqs. (2.5) and (2.17), and ignoring the $x$-dependent interference terms, we obtain

$$
\begin{gathered}
\tilde{S}^{-}\left(X, X^{\prime}\right)=\frac{i}{2} \sum_{n \in \Omega_{3}}\left[+\psi_{n}(X)+\bar{\psi}_{n}\left(X^{\prime}\right)\right. \\
\left.\quad+-\psi_{n}(X)-\bar{\psi}_{n}\left(X^{\prime}\right)\right], \quad x, x^{\prime} \in S_{\mathrm{L}}, \\
\tilde{S}^{+}\left(X, X^{\prime}\right)=\frac{i}{2} \sum_{n \in \Omega_{3}}\left[{ }^{+} \psi_{n}(X)^{+} \bar{\psi}_{n}\left(X^{\prime}\right)\right. \\
\left.\quad+{ }^{-} \psi_{n}(X)^{-} \bar{\psi}_{n}\left(X^{\prime}\right)\right], \quad x, x^{\prime} \in S_{\mathrm{R}} .
\end{gathered}
$$

Since singular functions (2.33) are constructed with the help of solutions of the Dirac equation for free particles (in the regions $S_{\mathrm{L}}$ and $S_{\mathrm{R}}$, respectively), they do not contribute to the fluxes under consideration. Thus,

$$
\left\langle J^{1}(x)\right\rangle^{c}=0,\left\langle T^{10}(x)\right\rangle^{c}=0, \quad x \in S_{\mathrm{L}} \text { or } S_{\mathrm{R}},
$$

which means that the fluxes calculated with the help of $S_{\text {in }}^{c}$ and $S^{p}$ coincide.

The quantities $\left\langle J^{0}(x)\right\rangle_{\text {in }}$ and $\left\langle T^{\mu \mu}(x)\right\rangle_{\text {in }}$ at $x \in S_{\mathrm{L} / \mathrm{R}}$ have to be renormalized. The procedure below has not been considered previously in QED with $x$-electric steps. Let us dis- cuss contributions to the quantities $\left\langle J^{0}(x)\right\rangle^{c}$ and $\left\langle T^{\mu \mu}(x)\right\rangle^{c}$ that are stipulated by the singular functions (2.33) at the regions $S_{\mathrm{L}}$ and $S_{\mathrm{R}}$. First we note that the definition of the range $\Omega_{3}$, given by Eq. (2.2), does not depend on variations of the electric field in the region $S_{\text {int }}$ which leave unchanged the potentials $U_{\mathrm{L}}$ and $U_{\mathrm{R}}$ - and therefore neither do the singular functions under consideration. These contributions from the left and from the right are different; this difference is due to different kinetic energies $\pi_{0}(\mathrm{~L} / \mathrm{R})$ on the left of the area $S_{\text {int }}$ and on the right of the area $S_{\text {int }}$. The quantities $\pi_{0}(\mathrm{~L} / \mathrm{R})$ are defined by potentials $U_{\mathrm{L}}$ and $U_{\mathrm{R}}$, respectively. The difference of these potentials gives us the magnitude of the $x$-step, $\pi_{0}(\mathrm{~L})-\pi_{0}(\mathrm{R})=U_{\mathrm{R}}-U_{\mathrm{L}}=\Delta U$, which defines the Klein range $\Omega_{3}$. It is clear that the difference $\Delta U$ is produced by work done by the electric field in the area $S_{\text {int }}$. However, it should be borne in mind that this work can be done by an electric field of any form and any intensity, including an arbitrarily low intensity, if the area $S_{\text {int }}$ is wide enough. Exactly in such a way the inhomogeneous structure of the vacuum in the presence of an $x$-step out of the field area $S_{\text {int }}$ is manifested. This effect is similar to a vacuum effect in the space around a magnetic flux confined inside a solenoid, i.e., in the presence of an Aharonov-Bohm potential. The mean numbers of pairs created from the vacuum, $N_{n}^{\text {cr }}$, are not determined by the magnitude $\Delta U$, but by the electric field intensity $E(x)$, so that for small fields, $E(x) \rightarrow 0$, these quantities are negligible, $N_{n}^{\text {cr }} \rightarrow 0$. Thus, nonzero values of the densities $\left\langle J^{0}(x)\right\rangle^{c}$ and $\left\langle T^{\mu \mu}(x)\right\rangle^{c}$ out of the area $S_{\text {int }}$ have nothing to do with real particles and with the vacuum polarization due to the fact that the electric field is absent there. These terms represent unobservable quasilocal contributions to the vacuum of free particles and they should be neglected in the spirit of the idea of renormalization.

Thus, we believe that the means $\left\langle J^{0}(x)\right\rangle_{\text {in }}$ and $\left\langle T^{\mu \mu}(x)\right\rangle_{\text {in }}$ at $x \in S_{\mathrm{L} / \mathrm{R}}$ have to be renormalized to the ones $\left\langle\left. J^{0}(x)\right|_{\text {in }} ^{\text {ren }}\right.$ and $\left\langle T^{\mu \mu}(x)\right\rangle_{\text {in }}^{\text {ren }}$ as follows:

$$
\begin{aligned}
& \left\langle J^{0}(x)\right\rangle_{\text {in }}^{\text {ren }}=\left\langle J^{0}(x)\right\rangle_{\text {in }}-\left\langle J^{0}(x)\right\rangle^{c}=\left\langle J^{0}(x)\right\rangle^{p}, \\
& \left\langle T^{\mu \mu}(x)\right\rangle_{\text {in }}^{\text {ren }}=\left\langle T^{\mu \mu}(x)\right\rangle_{\text {in }}-\left\langle T^{\mu \mu}(x)\right\rangle^{c}=\left\langle T^{\mu \mu}(x)\right\rangle^{p} .
\end{aligned}
$$

Note that fluxes (2.25) and (2.26) can be represented in the same manner with account taken of Eqs. (2.34). Using Eqs. (2.5), we find

$$
\begin{aligned}
& \left\langle J^{0}(x)\right\rangle^{p}= \begin{cases}-\bar{J}^{0}(\mathrm{~L}), & x \in S_{\mathrm{L}}, \\
\bar{J}^{0}(\mathrm{R}), & x \in S_{\mathrm{R}},\end{cases} \\
& \bar{J}^{0}(\mathrm{~L} / \mathrm{R})=\frac{e}{2} \sum_{n \in \Omega_{3}} j_{n}^{0}(\mathrm{~L} / \mathrm{R}), \quad j_{n}^{0}(\mathrm{~L} / \mathrm{R})=j_{n}^{1} / v^{\mathrm{L} / \mathrm{R}},
\end{aligned}
$$


where $j_{n}^{1}$ is given by Eq. (2.30), and

$$
\begin{aligned}
& \left\langle T^{00}(x)\right\rangle^{p}= \begin{cases}\frac{1}{2} \sum_{n \in \Omega_{3}} j_{n}^{0}(\mathrm{~L}) \pi_{0}(\mathrm{~L}), & x \in S_{\mathrm{L}}, \\
\frac{1}{2} \sum_{n \in \Omega_{3}} j_{n}^{0}(\mathrm{R})\left|\pi_{0}(\mathrm{R})\right|, & x \in S_{\mathrm{R}},\end{cases} \\
& \left\langle\left. T^{11}(x)\right|^{p}= \begin{cases}\frac{1}{2} \sum_{n \in \Omega_{3}} j_{n}^{1}\left|p^{\mathrm{L}}\right|, & x \in S_{\mathrm{L}}, \\
\frac{1}{2} \sum_{n \in \Omega_{3}} j_{n}^{1}\left|p^{\mathrm{R}}\right|, & x \in S_{\mathrm{R}},\end{cases} \right. \\
& \left\langle T^{k k}(x)\right\rangle^{p}= \begin{cases}\frac{1}{2} \sum_{n \in \Omega_{3}} j_{n}^{1}\left(p_{k}\right)^{2} /\left|p^{\mathrm{L}}\right|, & x \in S_{\mathrm{L}}, \\
\frac{1}{2} \sum_{n \in \Omega_{3}} j_{n}^{1}\left(p_{k}\right)^{2} /\left|p^{\mathrm{R}}\right|, & x \in S_{\mathrm{R}},\end{cases}
\end{aligned}
$$

Next, we discuss how it is possible to extract physically meaningful information from the obtained formal expressions (2.36) and (2.37). Let us calculate the charge density and the longitudinal current density of the particles created. To this end we turn to Eq. (2.30). We see that the created electrons with a given $n$ are moving to the left with the velocity $v^{\mathrm{L}}$, whereas the current density $e j_{n}^{1}$ is moving to the right. During the time $T$ these electrons carry the charge $e j_{n}^{1} T$ over a unit area $V_{\perp}$ of the surface $x=x_{\mathrm{L}}$. Taking into account that this charge is evenly distributed over the cylindrical volume of the length $v^{\mathrm{L}} T$, we see that the charge density of the created electrons with given $n$ is $e j_{n}^{1} /\left(-v^{\mathrm{L}}\right)$. Note that this quantity coincides with the density $-e j_{n}^{0}(\mathrm{~L})$, where $j_{n}^{0}(\mathrm{~L})$ is given by Eq. (2.36), $-e j_{n}^{0}(\mathrm{~L})=e j_{n}^{1} /\left(-v^{\mathrm{L}}\right)$. The created positrons with a given $n$ are moving to the right with the velocity $v^{\mathrm{R}}$, the current density $e j_{n}^{1}$ moves in the same direction. During the time $T$ these positrons carry the same charge $e j_{n}^{1} T$ over a unit area $V_{\perp}$ of the surface $x=x_{\mathrm{R}}$. This charge is evenly distributed over the cylindrical volume of the length $v^{\mathrm{R}} T$. Thus, the charge density of the created positrons with a given $n$ is $e j_{n}^{1} / v^{\mathrm{R}}$. This quantity coincides with the density $e j_{n}^{0}(\mathrm{R})$, where $j_{n}^{0}(\mathrm{R})$ is given by Eq. (2.36), $e j_{n}^{0}(\mathrm{R})=e j_{n}^{1} / v^{\mathrm{R}}$. This means that the charge density of the created pairs is

$J_{\mathrm{cr}}^{0}(x)= \begin{cases}-e \sum_{n \in \Omega_{3}} j_{n}^{0}(\mathrm{~L}), & x \in S_{\mathrm{L}}, \\ e \sum_{n \in \Omega_{3}} j_{n}^{0}(\mathrm{R}), & x \in S_{\mathrm{R}} .\end{cases}$

Comparing Eqs. (2.36) and (2.38) we can relate the quantity $J_{\mathrm{cr}}^{0}(x)$ with the one $\left\langle J^{0}(x)\right\rangle^{p}$. One can easily see that $J_{\mathrm{cr}}^{0}(x)$ and $J_{\mathrm{cr}}^{1}$ are two nonzero components of the $d$ dimensional Lorentz vector $J_{\mathrm{cr}}^{\mu}(x)$ (note that its transversal components are zero) that represents the current density of the created pairs,

$$
\begin{array}{ll}
J_{\mathrm{cr}}^{0}(x)=2\left\langle J^{0}(x)\right\rangle^{p}, & J_{\mathrm{cr}}^{k}(x)=2\left\langle J^{k}(x)\right\rangle^{p}=0 \quad \text { if } k \neq 1 \\
J_{\mathrm{cr}}^{1}(x)=2\left\langle J^{1}(x)\right\rangle^{p}, & x \in S_{\mathrm{L}} \text { or } S_{\mathrm{R}} .
\end{array}
$$

In the same manner, one can derive the following relations for the nonzero components of EMT:

$$
\begin{aligned}
T_{\mathrm{cr}}^{\mu \mu}(x) & =2\left\langle T^{\mu \mu}(x)\right\rangle^{p} \\
T_{\mathrm{cr}}^{10}(x) & =2\left\langle T^{10}(x)\right\rangle^{p}, x \in S_{\mathrm{L}} \text { or } S_{\mathrm{R}} .
\end{aligned}
$$

Thus, Eqs. (2.39) and (2.40) give the opportunity to derive all the characteristics of the created particles with the help of the function $S^{p}$ given by Eq. (2.22). Note that the physical quantities related to the particles created in the areas $S_{\mathrm{L}}$ and $S_{\mathrm{R}}$ satisfy the relations

$J_{\mathrm{cr}}^{0}\left(x_{\mathrm{L}}\right)=-J_{\mathrm{cr}}^{0}\left(x_{\mathrm{R}}\right), \quad J_{\mathrm{cr}}^{1}\left(x_{\mathrm{L}}\right)=J_{\mathrm{cr}}^{1}\left(x_{\mathrm{R}}\right)$,

$T_{\mathrm{cr}}^{\mu \mu}\left(x_{\mathrm{L}}\right)=T_{\mathrm{cr}}^{\mu \mu}\left(x_{\mathrm{R}}\right), \quad T_{\mathrm{cr}}^{10}\left(x_{\mathrm{L}}\right)=-T_{\mathrm{cr}}^{10}\left(x_{\mathrm{R}}\right)$.

Thus, in QED with critical $x$-step, there exist relations between the nonzero physical quantities (2.39), (2.40) and the in-in (out-out) mean values (2.32) and (2.35),

$J_{\mathrm{cr}}^{0}(x)=\left\langle J^{0}(x)\right\rangle_{\text {in }}^{\text {ren }}+\left\langle J^{0}(x)\right\rangle_{\text {out }}^{\text {ren }}$,

$J_{\mathrm{cr}}^{1}(x)=\left\langle J^{1}(x)\right\rangle_{\text {in }}-\left\langle J^{1}(x)\right\rangle_{\text {out }}$;

$T_{\mathrm{cr}}^{\mu \mu}(x)=\left\langle T^{\mu \mu}(x)\right\rangle_{\text {in }}^{\text {ren }}+\left\langle T^{\mu \mu}(x)\right\rangle_{\text {out }}^{\text {ren }}$,

$T_{\mathrm{cr}}^{10}(x)=\left\langle T^{10}(x)\right\rangle_{\text {in }}-\left\langle T^{10}(x)\right\rangle_{\text {out }}, \quad x \in S_{\mathrm{L}}$ or $S_{\mathrm{R}}$.

We see, for example, that $\left\langle\left. J^{0}(x)\right|_{\text {in }} ^{\text {ren }}\right.$ and $\left\langle J^{1}(x)\right\rangle_{\text {in }}$ are two nonzero components of the $d$ dimensional Lorentz vector $\left\langle J^{\mu}(x)\right\rangle_{\text {in }}^{\text {ren }}=\left\langle J^{\mu}(x)\right\rangle^{p}$, whereas $\left\langle J^{0}(x)\right\rangle_{\text {out }}^{\text {ren }}$ and - $\left\langle J^{1}(x)\right\rangle_{\text {out }}$ are two nonzero components of the same Lorentz vector. A similar relation holds for the vacuum means of EMT. We stress that the calculated physical quantities (2.42) are determined by precisely the final particles. Thus, the proposed procedure allows one to relate the characteristics of the created particles with quasilocal quantities.

\section{Back-reaction and consistency conditions}

The regularization and renormalization procedures discussed above allow you to expand the range of tasks to which QED with $x$-steps concentrated in restricted space areas can be applied. In such a way we study below the effects of the back-reaction on the vacuum instability, which allows one, in turn, to establish the so-called consistency conditions. In high-energy physics problems it is usually assumed that just from the beginning there exists a classical electric field having a given energy. The system of fermions interacting with this field is closed, that is, the total energy of the system is conserved. In the following we work under such an assumption. $^{5}$

\footnotetext{
5 One can, however, imagine an alternative situation when these charges are getting out of the regions $S_{\mathrm{L}}$ and $S_{\mathrm{R}}$ with the help of the work done by an external storage battery. For example, dealing with graphene devices, it is natural to assume that the constant strength on the graphene plane is due to the applied fixed voltage, i.e., we are dealing with an open system of fermions interacting with a classical electromagnetic field. In that case there would be no back-reaction problem. Note that the evolution of the mean electromagnetic field in the graphene, taking into account the back-reaction of the matter field to the applied timedependent external field, was considered in Ref. [34].
} 
Let us consider a volume $V=V_{\perp}\left(x_{\mathrm{R}}-x_{\mathrm{L}}\right)$, which contains the area $S_{\mathrm{int}}=\left(x_{\mathrm{L}}, x_{\mathrm{R}}\right)$. The total energy of the created particles in the volume $V$ is given by the corresponding volume integral of the energy density $T_{\mathrm{cr}}^{00}(t, x)$. The corresponding energy conservation reads

$$
\frac{\partial}{\partial t} \int_{V_{\perp}} \mathrm{d} \mathbf{r}_{\perp} \int_{x_{\mathrm{L}}}^{x_{\mathrm{R}}} T_{\mathrm{cr}}^{00}(t, x) \mathrm{d} x=-\oint_{\Sigma} T_{\mathrm{cr}}^{k 0}(x) \mathrm{d} f_{k},
$$

where $\Sigma$ is a $d-1$ dimensional surface surrounding the volume $V$ and $\mathrm{d} f_{k}, k=1, \ldots, d-1$, are the components of the surface element $d \mathbf{f}$. Taking into account that $T_{\mathrm{cr}}^{00}(t, x)$ in Eq. (3.1) does not depend on transversal coordinates, $T_{\mathrm{cr}}^{k 0}(x)=0$ for $k \neq 1$ and using Eqs. (2.30) and (2.31) we find that the rate of the energy density change of the created particles in the area $S_{\text {int }}$ per unit of the spatial volume of the hypersurface orthogonal to the $x$-direction is

$$
\frac{\partial}{\partial t} \int_{x_{\mathrm{L}}}^{x_{\mathrm{R}}} T_{\mathrm{cr}}^{00}(t, x) \mathrm{d} x=T_{\mathrm{cr}}^{10}(\mathrm{~L})-T_{\mathrm{cr}}^{10}(\mathrm{R})=-\Delta U \frac{N^{\mathrm{cr}}}{T V_{\perp}},
$$

where $N^{\mathrm{cr}}$, given by Eq. (2.14), is the total number of pairs created from the vacuum. It characterizes the loss of the energy that created particles carry away from the region $S_{\text {int }}$. At the same time, the constant rate (3.2) determines the power of the constant electric field spent on the pair creation. Integrating this rate over the time duration of an electric field from $t_{\text {in }}$ to $t_{\text {out }}$, and using the notation

$\Delta T_{\mathrm{cr}}^{00}(x)=-\int_{t_{\mathrm{in}}}^{t_{\mathrm{out}}} \frac{\partial}{\partial t} T_{\mathrm{cr}}^{00}(t, x) \mathrm{d} t$,

we find the total energy density of created pairs per unit of the orthogonal hypersurface as

$\int_{x_{\mathrm{L}}}^{x_{\mathrm{R}}} \Delta T_{\mathrm{cr}}^{00}(x) \mathrm{d} x=\Delta U \frac{N^{\mathrm{cr}}}{V_{\perp}}$.

We assume that the constant external electric field which represents the $x$-step exists during a macroscopically large time period $T$ satisfying the condition (1.4).

Now we are able to discuss the back-reaction problem. It is clear that while creating pairs from the vacuum the external electric field is losing its energy and should change (weaken) with time. Thus, the applicability of the constant field approximation, which is used in the formulation of QED with $x$-step, is limited by the smallness of the back-reaction. The results obtained above allow us to find conditions that provide this smallness; we call these consistency conditions in what follows. These conditions can be obtained from the requirement that the energy density (3.3) is essentially smaller than the energy density of the external electric field (per unit of the orthogonal hypersurface).

To do this, we consider the case of an uniform electric field confined between two capacitor plates separated by a finite distance $L$. We call such a configuration an $L$-constant electric field. In this case $\Delta U=e E L$. The particle creation in such a case was studied in Ref. [27]. If

$L \gg(e E)^{-1 / 2} \max \left\{1, m^{2} / e E\right\}$,

the $L$-constant electric field can reasonably be expected to simulate a small-gradient electric field. Besides, in such a case boundary effects are negligible and the density $\Delta T_{\mathrm{cr}}^{00}(x)$ is uniform in the leading term approximation, $\Delta T_{\mathrm{cr}}^{00}(x)=$ $\Delta T_{\mathrm{cr}}^{00}$. In this case the number density in the RHS of Eq. (3.3) has the form

$\frac{N^{\mathrm{cr}}}{V_{\perp}}=L n^{\mathrm{cr}}, \quad n^{\mathrm{cr}}=T r^{\mathrm{cr}}, \quad r^{\mathrm{cr}}=\frac{J(e E)^{d / 2}}{(2 \pi)^{d-1}} \exp \left(-\pi \frac{m^{2}}{e E}\right)$,

where $n^{\text {cr }}$ is the number density of pairs created per unit volume of the field area and $J=2^{[d / 2]-1}$ is the number of spin degrees of freedom ( $J=1$ for scalar particles) [27]. Taking into account that $x_{\mathrm{R}}-x_{\mathrm{L}}=L$ and integrating over $x$ in Eq. (3.3) we find that the total energy density of created pairs per the volume unit is

$\Delta T_{\mathrm{cr}}^{00}=e E L T r^{\mathrm{cr}}$.

Note that the density $r^{\mathrm{cr}}$, given by Eq. (3.5), can be identified with the pair-production rate in the case of a constant uniform external electric field; see Ref. [21]. The number density $n^{\mathrm{cr}}$, given by Eq. (3.5), coincides with result obtained for the case of the $T$-constant field (a uniform electric field which effectively acts during a sufficiently large but finite time interval T) [21].

Note that unlike the case of the pair creation by the $T$ constant field, considered in the framework of QED with $t$-step, where $N^{\mathrm{cr}}$ is a linear function of the finite time duration of the field $T$ and $L \rightarrow \infty$ is a given constant, $N^{\text {cr }}$ given by Eq. (3.5) is a linear function of the field length $L$ and $T \rightarrow \infty$ is a given constant. The $T$-constant and $L$ constant fields are physically distinct. Note that while in the $T$-constant field model it is allowed that $L / T \sim 1$, in the case under consideration (of a finite field area) we assumed that Eq. (2.4) is satisfied. The latter assumption implies the inequality $T \gg L$. If we use the fact that the pair-production rate $r^{\mathrm{cr}}$, given by Eq. (3.5), is the same both for $T$-constant and $L$-constant fields, then we can compare energy densities of created pairs per unit volume for both cases. The energy density of the created pairs $\left\langle T_{00}\left(t_{\text {out }}\right)\right\rangle$ per unit volume for the case of $T$-constant field was derived in Refs. [34-36] and it has the form

$\left\langle T_{00}\left(t_{\text {out }}\right)\right\rangle=e E T^{2} r^{\mathrm{cr}}$.

The densities (3.6) and (3.7) are related as follows:

$\Delta T_{\mathrm{cr}}^{00}=\frac{L}{T}\left\langle T_{00}\left(t_{\mathrm{out}}\right)\right\rangle$, 
which means that $\Delta T_{\mathrm{cr}}^{00} \ll\left\langle T_{00}\left(t_{\text {out }}\right)\right\rangle$. Thus, taking into account that the area occupied by the external field is finite significantly reduces the energy densities of the created pairs per unit volume for given field strength $E$ and its duration $T$. In turn, this also affects the degree of the back-reaction.

In problems of high-energy physics it is usually assumed that just from the beginning there exists a classical electric field having a given energy density. The system of fermions interacting with this field is supposed to be closed, that is, its total energy is conserved. Such an assumption is consistent only if the back-reaction due to the pair creation is relatively small with respect to the background. For the same reasons QED with either strong $T$-constant or $L$-constant fields can be considered as a consistent model only if the back-reaction is small. Below we derive the conditions which provide such a smallness for QED with $x$-step. Similar to the case of QED with $t$-step (see Ref. [28]), we call them consistency conditions.

The consistency conditions follow from the supposition that the energy density $\Delta T_{\mathrm{cr}}^{00}$, arising precisely due to the action of a $L$-constant electric field, should be essentially smaller than the energy density of the external electric field. As such a density in $d=4$ dimensions we take the classical energy density $\mathcal{E}^{(0)}$ of the electric field, $\mathcal{E}^{(0)}=E^{2} / 8 \pi$. Thus, the condition of the smallness of the back-reaction can be written as $\Delta T_{\mathrm{cr}}^{00} \ll E^{2} / 8 \pi$. Taking into account Eq. (3.6), we obtain the sought-for consistency conditions as a restriction from above on the dimensionless parameter $e E L T$ :

$e E L T \ll \frac{\pi^{2}}{J \alpha} \exp \left(\pi \frac{m^{2}}{e E}\right)$.

Here $\alpha$ is the fine structure constant, $\alpha=e^{2} / c \hbar=e^{2}$, and $J=2$.

Note that using the appropriate number of the spin degrees of freedom inequality (3.9) can be generalized for scalar particles $(J=1)$ and for vector particles $(J=3)$. On the other hand, all the asymptotic formulas have been obtained under conditions (1.4) and (3.4), which impose restrictions on the parameter $e E L T$ from below,

$e E L T \gg \max \left\{1,\left(m^{2} / e E\right)^{2}\right\}$.

One can easily extend these results to $d$ dimensions, using the expressions for $r^{\mathrm{cr}}$, given by Eq. (3.5).

Since $\pi^{2} / J \alpha \gg 1$, there exists a range of values of parameters $E, L$ and $T$ that satisfies both inequalities. We recall once again that the consistency conditions in the case of QED with $t$-step (specifically for the case of the $T$-constant field) were obtained in Ref. [35,36]. They have the following form:

$$
\max \left\{1,\left(m^{2} / e E\right)^{2}\right\} \ll e E T^{2} \ll \frac{\pi^{2}}{J \alpha} \exp \left(\pi \frac{m^{2}}{e E}\right) .
$$

Taking into account that in the case under consideration the dimensionless parameter satisfies the inequality $e E L T \ll e E T^{2}$, one can see that the consistency condition (3.9) is much less restrictive than the one of (3.11).

\section{Brief summary}

We consider the present article as a natural and important addition to the nonperturbative formulation of QED with $x$ steps presented in Ref. [20]. There, we have calculated global effects (global quantities) of zeroth order with respect to the radiative interaction, as regards the vacuum-to-vacuum transition amplitude, mean differential and total numbers of created particles, and so on. Here we propose a new renormalization and volume regularization procedures which allow one to calculate and distinguish physical parts of different matrix elements of the operators of the current and of the energymomentum tensor, at the same time relating the latter with characteristics of the vacuum instability. The renormalization and volume regularization procedures are associated with the introduction of a modified inner product and a parameter $\tau$ of the regularization. Based on physical considerations, we fix this parameter. It turns out that in the Klein range this parameter can be interpreted as the time of the observation of the pair-production process. We derive conditions that provide the time independence of the introduced inner product.

With the help of the calculated characteristics of the vacuum instability, we consider the problem of the back-reaction problem in QED with $x$-steps. In the case of an uniform electric field confined between two capacitor plates separated by a finite distance $L$, we see that the smallness of the backreaction implies a restriction on the dimensionless parameter $e E L T$. We see this consistency condition is much less restrictive than the one derived for QED with $t$-steps in our work [28].

It should be noted that, recently, there was proposed a new formulation of locally constant field approximations (LCFAs) in QED with $x$-steps, which does not rely on the Heisenberg-Euler action [31] (a similar approximation was formulated in QED with $t$-steps slowly varying with time [37]). As part of this formulation, we have constructed universal approximate representations for the total number and current density of the created particles in arbitrary weakly inhomogeneous $x$-steps. We hope that the regularization and renormalization procedures proposed in the present article will allow us to formulate soon a LCFA adequate for calculating the vacuum means of the current density and EMT in QED with arbitrary weakly inhomogeneous $x$-steps.

Acknowledgements The work of both authors is supported by the Russian Science Foundation (Grant no. 19-12-00042). 
Data Availability Statement This manuscript has associated data in a data repository. [Authors' comment: There is no data associated with this work due to its theoretical content.]

Open Access This article is licensed under a Creative Commons Attribution 4.0 International License, which permits use, sharing, adaptation, distribution and reproduction in any medium or format, as long as you give appropriate credit to the original author(s) and the source, provide a link to the Creative Commons licence, and indicate if changes were made. The images or other third party material in this article are included in the article's Creative Commons licence, unless indicated otherwise in a credit line to the material. If material is not included in the article's Creative Commons licence and your intended use is not permitted by statutory regulation or exceeds the permitted use, you will need to obtain permission directly from the copyright holder. To view a copy of this licence, visit http://creativecomm ons.org/licenses/by/4.0/.

Funded by SCOAP ${ }^{3}$.

\section{Appendix A: Some properties of solutions of the Dirac equation with critical $x$-steps}

Here we briefly recall some features of the solutions of the Dirac equation with critical $x$-steps established in Ref. [20] (see Sect. III and Appendix B).

The Dirac equation with a $x$-steps has the form

$i \partial_{0} \psi(X)=\hat{H} \psi(X)$,

$\hat{H}=\gamma^{0}\left(-i \gamma^{j} \partial_{j}+m\right)+U(x), \quad j=1, \ldots D$,

and its solutions are of the form

$$
\begin{aligned}
& \psi(X)=\exp \left(-i p_{0} t+i \mathbf{p}_{\perp} \mathbf{r}_{\perp}\right) \psi_{n}(x), \mathbf{p}_{\perp}=\left(p^{2}, \ldots, p^{D}\right), \\
& \psi_{n}(x)=\left\{\gamma^{0}\left[p_{0}-U(x)\right]-\gamma^{1} \hat{p}_{1}-\gamma_{\perp} \mathbf{p}_{\perp}+m\right\} \varphi_{n}^{(\chi)}(x) v_{\chi}, \\
& \gamma^{0} \gamma^{1} v_{\chi}=\chi v_{\chi}, \quad \chi= \pm 1, \quad v_{\chi^{\prime}, \sigma^{\prime}}^{\dagger} v_{\chi, \sigma}=\delta_{\chi^{\prime} \chi} \delta_{\sigma^{\prime} \sigma} .
\end{aligned}
$$

Here $\psi(x)$ is a $2^{[d / 2]}$-component spinor, $[d / 2]$ stands for the integer part of $d / 2, \gamma^{\mu}$ are the $\gamma$-matrices in $d$ dimensions, and $v_{\chi, \sigma}, \sigma=\left(\sigma_{1}, \sigma_{2}, \ldots, \sigma_{[d / 2]-1}\right), \sigma_{s}= \pm 1$, is a set of constant orthonormalized spinors. Scalar functions $\varphi_{n}^{(\chi)}(x)$ satisfy the following second-order differential equation:

$$
\left\{\hat{p}_{x}^{2}-i \chi U^{\prime}(x)-\left[p_{0}-U(x)\right]^{2}+\mathbf{p}_{\perp}^{2}+m^{2}\right\} \varphi_{n}^{(x)}(x)=0 .
$$

These solutions are parametrized by the set of quantum numbers $n=\left(p_{0}, \mathbf{p}_{\perp}, \sigma\right)$. To construct complete sets necessary for our purposes it is sufficient to choose only one value of $\chi$. Furthermore, for convenience, we choose a specific fixation, and then do not use this index at all, $\varphi_{n}^{(\mathrm{x})}(x)=\varphi_{n}(x)$.

We construct two types of complete sets of solution in the form (A2). The first one, $\zeta \psi_{n}(X)$, is defined by the functions $\varphi_{n}(x)$ denoted ${ }_{\zeta} \varphi_{n}(x)$ and the second one, ${ }^{\zeta} \psi_{n}(X)$, is defined by functions $\varphi_{n}(x)$ denoted ${ }^{\zeta} \varphi_{n}(x)$. Asymptotically, these functions have the forms

$$
\begin{array}{ll}
{ }_{\zeta} \varphi_{n}(X)={ }_{\zeta} \mathcal{N} \exp \left[i p^{\mathrm{L}}\left(x-x_{\mathrm{L}}\right)\right], & x \in S_{\mathrm{L}}, \\
{ }^{\zeta} \varphi_{n}(X)=\zeta \mathcal{N} \exp \left[i p^{\mathrm{R}}\left(x-x_{\mathrm{R}}\right)\right], & x \in S_{\mathrm{R}},
\end{array}
$$

where $p^{\mathrm{L}}$ and $p^{\mathrm{R}}$ are given by Eq. (2.1). Thus, asymptotically the solutions $\zeta \psi_{n}(X)$ and ${ }^{\zeta} \psi_{n}(X)$ describe particles with given real momenta $p^{\mathrm{L} / \mathrm{R}}$ along the $x$ axis. Note that if $U_{\mathrm{R}}=-U_{\mathrm{L}}$ then there is a symmetry $\left|\pi_{0}(\mathrm{R})\right|=$ $\left|\pi_{0}(\mathrm{~L})\right|_{p_{0} \rightarrow-p_{0}}$ with respect to the change $p_{0} \rightarrow-p_{0}$. The solutions $\zeta \psi_{n}(X)$ and ${ }^{\zeta} \psi_{n}(X)$ are orthonormalized with respect to the inner product on the $x=$ const hyperplane,

$$
\begin{aligned}
& \left({ }_{\zeta} \psi_{n}, \zeta^{\prime} \psi_{n^{\prime}}\right)_{x}=\zeta \eta_{\mathrm{L}} \delta_{\zeta, \zeta^{\prime}} \delta_{n, n^{\prime}}, \quad \eta_{\mathrm{L}}=\operatorname{sgn} \pi_{0}(\mathrm{~L}), \\
& \left({ }^{\zeta} \psi_{n}, \zeta^{\zeta^{\prime}} \psi_{n^{\prime}}\right)_{x}=\zeta \eta_{\mathrm{R}} \delta_{\zeta, \zeta^{\prime}} \delta_{n, n^{\prime}}, \quad \eta_{\mathrm{R}}=\operatorname{sgn} \pi_{0}(\mathrm{R}) ; \\
& \left(\psi, \psi^{\prime}\right)_{x}=\int \psi^{\dagger}(X) \gamma^{0} \gamma^{1} \psi^{\prime}(X) \mathrm{d} t \mathrm{~d} \mathbf{r}_{\perp},
\end{aligned}
$$

if

$$
\begin{aligned}
& { }^{\zeta} \mathcal{N}={ }^{\zeta} C Y,{ }_{\zeta} \mathcal{N}={ }_{\zeta} C Y, Y=\left(V_{\perp} T\right)^{-1 / 2}, \\
& { }^{\zeta} C=\left[2\left|p^{\mathrm{R}}\right|\left|\pi_{0}(\mathrm{R})-\chi p^{\mathrm{R}}\right|\right]^{-1 / 2}, \\
& { }_{\zeta} C=\left[2\left|p^{\mathrm{L}}\right|\left|\pi_{0}(\mathrm{~L})-\chi p^{\mathrm{L}}\right|\right]^{-1 / 2} .
\end{aligned}
$$

We consider our theory in a large space-time box that has a spatial volume $V_{\perp}=\prod_{j=2}^{D} K_{j}$ and the time dimension $T$, where all $K_{j}$ and $T$ are macroscopically large. It is supposed that all the solutions $\psi(X)$ are periodic under transitions from one box to another. Then the integration in (A5) over the transverse coordinates is fulfilled from $-K_{j} / 2$ to $+K_{j} / 2$, and over the time $t$ from $-T / 2$ to $+T / 2$. Under these suppositions, the inner product (A5) does not depend on $x$.

It is assumed that each pair of solutions ${ }_{\zeta} \psi_{n}(X)$ and ${ }^{\zeta} \psi_{n}(X)$ with any $n \in \Omega_{1} \cup \Omega_{3} \cup \Omega_{5}$ is complete in the space of solutions with the corresponding $n$. Mutual decompositions of such solutions have the form (2.5). The decomposition coefficients $g$ have the following origin:

$$
\begin{aligned}
\left({ }_{\zeta} \psi_{n}, \zeta^{\prime} \psi_{n^{\prime}}\right)_{x} & =\delta_{n, n^{\prime}} g\left(\zeta \zeta^{\prime}\right), \\
g\left(\left.\zeta^{\prime}\right|_{\zeta}\right) & =g\left(\left.\zeta\right|^{\prime}\right)^{*}, \quad n \in \Omega_{1} \cup \Omega_{3} \cup \Omega_{5} .
\end{aligned}
$$

They satisfy the unitary relations

$$
\begin{aligned}
& \mid g\left(\left.\left(\left._{-}\right|^{+}\right)\right|^{2}=\left|g\left(++_{+}\right)\right|^{2}, \quad\left|g\left(\left._{+}\right|^{+}\right)\right|^{2}=\left|g\left(-\left.\right|^{-}\right)\right|^{2},\right. \\
& \frac{g\left(+\left.\right|^{-}\right)}{g\left(-\left.\right|^{-}\right)}=\frac{g\left(+I_{-}\right)}{g\left(\left.{ }^{+}\right|_{+}\right)},\left|g\left(+\left.\right|^{-}\right)\right|^{2}-\left|g\left(+\left.\right|^{+}\right)\right|^{2}=-\eta_{\mathrm{L}} \eta_{\mathrm{R}} .
\end{aligned}
$$

In the framework of a field theory an observable $F$ can be realized as an inner product of the type (2.3) of localizable 
wave packets $\psi(X)$ and $\hat{F} \psi^{\prime}(X)$,

$F\left(\psi, \psi^{\prime}\right)=\left(\psi, \hat{F} \psi^{\prime}\right)$,

where $\hat{F}$ is a differential operator and $\psi(X)$ and $\psi^{\prime}(X)$ are solutions of the Dirac equation. Assuming that an observable $F\left(\psi, \psi^{\prime}\right)$ is time independent during the time $T$ one can represent this observable in the following form of an average value over the period $T$ :

$\langle F\rangle=\frac{1}{T} \int_{-T / 2}^{+T / 2} F\left(\psi, \psi^{\prime}\right) \mathrm{d} t$.

In general the wave packets $\psi(X)$ and $\psi^{\prime}(X)$ can be decomposed into plane waves $\psi_{n}(X)$ and $\psi_{n}^{\prime}(X)$ with given $n$,

$\psi(X)=\sum_{n} \alpha_{n} \psi_{n}(X), \quad \psi^{\prime}(X)=\sum_{n} \alpha_{n}^{\prime} \psi_{n}^{\prime}(X)$,

where $\psi_{n}(X)$ and $\psi_{n}^{\prime}(X)$ are superpositions of the solution $\mathrm{s}_{\zeta} \psi_{n}(X)$ and ${ }^{\zeta} \psi_{n}(X)$. Taking into account the orthogonality relation (A5) one finds that the decomposition of $\langle F\rangle$ into plane waves with given $n$ does not contain interference terms,

$$
\langle F\rangle=\sum_{n} F\left(\alpha_{n} \psi_{n}, \alpha_{n}^{\prime} \psi_{n}^{\prime}\right)
$$

\section{References}

1. A.I. Nikishov, Pair production by a constant electric field. Zh. Eksp. Teor. Fiz. 57, 1210 (1969) [Transl. Sov. Phys. JETP 30, 660 (1970)]

2. A.I. Nikishov, Problems of intense external field in quantum electrodynamics in Quantum Electrodynamics of Phenomena in Intense Fields, Proc. P.N. Lebedev Phys. Inst., vol. 111 (Nauka, Moscow, 1979), p. 153

3. W. Greiner, B. Müller, J. Rafelski, Quantum Electrodynamics of Strong Fields (Springer, Berlin, 1985)

4. R. Ruffini, G. Vereshchagin, S. Xue, Electron-positron pairs in physics and astrophysics: from heavy nuclei to black holes. Phys. Rep. 487, 1 (2010)

5. F. Gelis, N. Tanji, Schwinger mechanism revisited. Prog. Part. Nucl. Phys. 87, 1 (2016)

6. D.M. Gitman, Processes of arbitrary order in quantum electrodynamics with a pair-creating external field. J. Phys. A 10, 2007 (1977)

7. E.S. Fradkin, D.M. Gitman, Furry picture for quantum electrodynamics with pair-creating external field. Fortschr. Phys. 29, 381 (1981)

8. E.S. Fradkin, D.M. Gitman, S.M. Shvartsman, Quantum Electrodynamics with Unstable Vacuum (Springer, Berlin, 1991)

9. O. Klein, Die Reflexion von Elektronen einem Potentialsprung nach der relativistischen Dynamik von Dirac. Z. Phys. 53, 157 (1929)

10. O. Klein, Elelrtrodynamik und Wellenmechanik vom Standpunkt des Korrespondenzprinzips. Z. Phys. 41, 407 (1927)

11. F. Sauter, Über das Verhalten eines Elektrons im homogenen elektrischen Feld nach der relativistischen Theorie Diracs. Z. Phys. 69, $742(1931)$

12. F. Sauter, Zum “Klenschen Paradoxon”. Z. Phys. 73, 547 (1931)
13. A.I. Nikishov, Barrier scattering in field theory: removal of Klein paradox. Nucl. Phys. B 21, 346 (1970)

14. A. Hansen, F. Ravndal, Klein's paradox and its resolution. Phys. Scr. 23, 1036 (1981)

15. A.I. Nikishov, Scattering and pair production by a potential barrier, Yad. Fiz. 67, 1503 (2004) [Transl. Phys. Atom. Nucl. 67, 14; On the theory of scalar pair production by a potential barrier, Problems of Atomic Science and Technology (Kharkov, Ukraine, 2001), p. 103 [arXiv:hep-th/0111137]

16. T. Damour, Klein paradox and vacuum polarization, in Proceedings of the First Marcel Grossmann Meeting on General Relativity, ed. by R. Ruffini, (North-Holland, Amsterdam, 1977), p. 459

17. R.-C. Wang, C.-Y. Wong, Finite-size effect in the Schwinger particle-production mechanism. Phys. Rev. D 38, 348 (1988)

18. N. Dombey, A. Calogeracos, Seventy years of the Klein paradox. Phys. Rep. 315, 41 (1999)

19. N. Dombey, A. Calogeracos, History and physics of the Klein paradox. Contemp. Phys. 40, 313 (1999). arXiv:quant-ph/9905076

20. S.P. Gavrilov, D.M. Gitman, Quantization of charged fields in the presence of critical potential steps. Phys. Rev. D. 93, 045002 (2016)

21. S.P. Gavrilov, D.M. Gitman, Vacuum instability in external fields. Phys. Rev. D 53, 7162 (1996)

22. T.C. Adorno, S.P. Gavrilov, D.M. Gitman, Exactly solvable cases in QED with t-electric potential steps. Int. J. Mod. Phys. A. 32, 1750105 (2017). arXiv:1512.01288

23. T.C. Adorno, S.P. Gavrilov, D.M. Gitman, Int. J. Mod. Phys. A 32, 1750105 (2017)

24. T.C. Adorno, S.P. Gavrilov, D.M. Gitman, Violation of vacuum stability by inverse square electric fields. Eur. Phys. J. C 78, 1021 (2018). arXiv:1807.01642

25. T.C. Adorno, R. Ferreira, S.P. Gavrilov, D.M. Gitman, Role of switching-on and -off effects in the vacuum instability. Int. J. Mod. Phys. A 33, 1850060 (2018)

26. Y. Kluger, E. Mottola, J.M. Eisenberg, Quantum Vlasov equation and its Markov limit. Phys. Rev. D 58, 125015 (1998)

27. S.P. Gavrilov, D.M. Gitman, Scattering and pair creation by a constant electric field between two capacitor plates. Phys. Rev. D 93, 045033 (2016)

28. S.P. Gavrilov, D.M. Gitman, Consistency restrictions on maximal electric field strength in QFT. Phys. Rev. Lett. 101, 130403 (2008)

29. S.P. Gavrilov, D.M. Gitman, A.A. Shishmarev, Particle scattering and vacuum instability by exponential steps. Phys. Rev. D 96, 096020 (2017). arXiv: 1709.06997

30. S.P. Gavrilov, D.M. Gitman, A.A. Shishmarev, States of charged quantum fields and their statistical properties in the presence of critical potential steps. Phys. Rev. A 99, 052116 (2019). arXiv: 1901.01217

31. S.P. Gavrilov, D.M. Gitman, A.A. Shishmarev, Pair production from the vacuum by weakly inhomogeneous x-electric potential steps. Phys. Rev. D. 99, 116014 (2019). arXiv:1903.05925

32. G. Plunien, B. Müller, W. Greiner, The Casimir effect. Phys. Rep. 134, 87 (1986)

33. D. Gitman, I. Tyutin, Quantization of Fields with Constraints (Springer, Berlin, 1990)

34. S.P. Gavrilov, D.M. Gitman, N. Yokomizo, Dirac fermions in strong electric field and quantum transport in graphene. Phys. Rev. D 86, $125022(2012)$

35. S.P. Gavrilov, Effective energy-momentum tensor of strong-field QED with unstable vacuum. J. Phys. A 39, 6407 (2006)

36. S.P. Gavrilov, D.M. Gitman, Energy-momentum tensor in thermal strong-field QED with unstable vacuum. Phys. Rev. D 78, 045017 (2008)

37. S.P. Gavrilov, D.M. Gitman, Vacuum instability in slowly varying electric fields. Phys. Rev. D 95, 076013 (2017) 